\title{
REDUCTION TO REAL INFINITESIMAL CHARACTER IN AFFINE HECKE ALGEBRAS
}

\author{
DAN BARBASCH AND ALLEN MOY
}

\section{INTRODUCTION}

The main result of this paper is to show that the problem of the determination of the unramified unitary dual of a split $p$-adic group is equivalent to the problem of determining the unitary dual of the corresponding graded Hecke algebra.

In [BM], the authors established this equivalence in the case of Iwahori spherical representations under a certain restriction; namely, it was essential for the infinitesimal character to be real (in the terminology of [BM]). In terms of the Langlands-Deligne-Lusztig parameters $(s, u, \rho)[\mathrm{KL}]$, the restriction is that $s \in{ }^{L} G$ be a purely hyperbolic element. The technique used in [BM] was to combine the notion of the signature of a $K$-character in [V] with some facts which follow from [KL], namely, that the $\mathscr{H}_{W}$-characters of tempered representations are linearly independent. This is essentially true precisely when the infinitesimal character is real; for if not, $s$ has an elliptic part $s_{e}$ such that ${ }^{L} G\left(s_{e}\right) \neq{ }^{L} G$. Then the $\mathscr{H}_{W}$-characters of tempered representations behave like induced characters from this smaller group and there is no a priori reason why they should be independent; in general, they are not.

The removal of the real infinitesimal character restriction is Theorem 8.1. The proof of Theorem 8.1 is entirely different from [BM]. What we prove, based on ideas of Lusztig [Ls2], is that a certain Jantzen type filtration for standard modules for $\mathscr{H}$ is equivalent to the same kind of filtration for a corresponding Hecke algebra $\mathscr{H}\left(s_{e}\right)$. In this equivalence, representations with infinitesimal characters with elliptic part $s_{e}$ correspond; but since $s_{e}$ is a central element in ${ }^{L} G\left(s_{e}\right)$, we are reduced to the setting of [BM].

We give a few more details. Consider the polar decomposition $s=s_{e} s_{h}$ of $s$ into its elliptic and hyperbolic parts. Let $\mathscr{C}(G, \mathscr{O})$ be the category of representations of $G$ of finite length and with infinitesimal character having elliptic part in the Weyl group orbit $\mathscr{O}$ of $s_{e}$. If $G$ has connected center, the centralizer $C_{L}\left(s_{e}\right)$ of $s_{e}$ in ${ }^{L} G$ is connected. Let $G^{\prime}$ be the split $p$-adic group whose $L$-group is $C_{L_{G}}\left(s_{e}\right)$. Then Theorem 4.3 states that there is a category equiva-

Received by the editors October 30, 1991 and, in revised form, July 9, 1992.

1991 Mathematics Subject Classification. Primary 22E50.

The authors were supported in part by NSF grants DMS-9104117 for Barbasch and DMS9014483 for Moy. 
lence between $\mathscr{C}(G, \mathscr{O})$ and $\mathscr{C}\left(G^{\prime},\left\{s_{e}\right\}\right)$. The category $\mathscr{C}\left(G^{\prime},\left\{s_{e}\right\}\right)$ in turn is naturally equivalent to $\mathscr{C}\left(G^{\prime},\{1\}\right)$, the category of representations with real infinitesimal character. The idea of the proof as well as the statement is due to Lusztig. In [Ls2], he first constructs a graded Hecke algebra $\mathbb{H}$ corresponding to the Hecke algebra $\mathscr{H}(G / / \mathscr{I})$ of Iwahori spherical functions. Then he shows for fixed matching infinitesimal characters $\chi$ and $\bar{\chi}$ that there is an isomorphism between $\mathscr{H}(G / / \mathscr{I})_{\chi}$ and $\mathbb{H}_{\bar{\chi}}$.

In order to apply these ideas to questions of unitarity we need two additional ingredients. The first is a slight generalization of Lusztig's methods to yield an isomorphism between $\mathscr{H}(G / / \mathscr{I})_{\chi}$ and $\mathbb{H}_{\bar{\chi}}$ in an analytic family setting. This is done in $\S 4$. The differences to [Ls2] are technical, but they are essential for

our argument. When $C_{L_{G}}\left(s_{e}\right)$ is a Levi subgroup of ${ }^{L} G$, the group $G^{\prime}$ is a Levi subgroup of $G$. In this case the equivalence of categories is effectively the irreducibility of certain unitarily induced representations from $G^{\prime}$ to $G$ and is of course much simpler. This is analogous to reduction to real infinitesimal character in real reductive groups [B, V]. However, the group $C_{L_{G}}\left(s_{e}\right)$ need not be a Levi subgroup. Here, the equivalence of categories between $\mathscr{C}(G, \mathscr{O})$ and $\mathscr{C}\left(G^{\prime}, 1\right)$ should be thought of as a strong realization, in a limited situation, of endoscopic transfer of representations between $G^{\prime}$ and $G$. The transfer has the important property that certain data, related to the signature character of a hermitian representation, are preserved.

The second ingredient needed for unitarity is defining a natural ${ }^{*}$ operation on the graded Hecke algebra $\mathbb{H}_{\mathscr{O}}$ and relating it to the ${ }^{*}$ operation on $\mathscr{H}(G / / \mathscr{F})$. This is done in $\S 5$. In $\S 8$, the unitarity of Iwahori spherical representations is reduced to the question of the determination of the unitary dual of a corresponding graded Hecke algebra at real infinitesimal character. In this respect Theorem 5.6 for the * operation is essential. We hope to pursue the problem of determining this dual in future work.

We also note Theorem 6.3 which basically describes the Hecke algebra module of an induced module in terms of the action of the affine part of the Hecke algebra. Undoubtedly this is known to the specialist.

Finally we comment on the validity of our results for the cases when $G$ is a nonsplit group. In this setting, the Hecke algebra with respect to an Iwahori subgroup is a Hecke algebra associated to a parameter set. The allowable parameter sets can be found in [T]. When these algebras are graded at the orbit $\mathscr{O}=\{1\}$, one obtains a graded Hecke algebra with parameter as described in [Ls3, §2.13]. For these graded Hecke algebras, it is known that there are only finitely many real tempered representations. The fact that the $W$ characters of these real tempered representations are linearly independent follows (presumably) from [Ls4] (generalization of [BM, Theorem 4.4]). Then the techniques of $[\mathrm{BM}]$ apply, so the unitarity of real representations for these groups can be detected on the Iwahori fixed vectors.

\section{REVIEW OF BASIC RESULTS}

We use the notation in [BM]. Fix a $p$-adic field $\mathbb{F}$, and let $G$ be the $\mathbb{F}$ rational points of a split reductive group. We do not require $G$ to be a specific 
type in its isogeny class. Thus, for example, in the extremes, $G$ can be adjoint or simply connected. The Hecke algebra $\mathscr{H}(G / / \mathscr{I})$ of compactly supported Iwahori-spherical functions has a vector space decomposition

$$
\mathscr{H}(G / / \mathscr{J})=\mathscr{H}_{K} \otimes \mathscr{A},
$$

where $\mathscr{H}_{K}$ and $\mathscr{A}$ are subalgebras of $\mathscr{H}(G / / \mathscr{I})$.

The subalgebra $\mathscr{H}_{K}=\mathscr{H}(K / / \mathscr{I})$ is the finite Hecke algebra of functions whose support lies in $K=G\left(R_{\mathbb{F}}\right)$, the integral points of $G$.

The subalgebra $\mathscr{A}$ is abelian. To describe it explicitly, choose a Borel subgroup $B=A N$ compatible with $K$. Let $\mathscr{X}=\operatorname{Hom}\left(G_{m}, A\right)$ be the algebraic homomorphisms of $G_{m}=\mathrm{GL}(1, \mathbb{F})$ into $A$. Also, let $\Pi \subseteq R^{+} \subseteq R \subseteq \mathscr{X}$ be the simple coroots, the positive coroots, and the coroots determined by $B$ and $A$ respectively. Similarly, let $\mathscr{Y}=\check{\mathscr{X}}=\operatorname{Hom}\left(A, G_{m}\right)$ and let $\check{\Pi} \subseteq \check{R}^{+} \subseteq \check{R} \subseteq \check{\mathscr{X}}$ be the simple roots, the positive roots, and the roots.

We mention for later use that ${ }^{L} G$ is the (connected) complex alegbraic group attached to the data $(\mathscr{Y}, \mathscr{X}, \check{R}, R, \check{\Pi})$ dual to $(\mathscr{X}, \mathscr{Y}, R, \check{R}, \Pi)$. In particular, ${ }^{L} A=\check{\mathscr{X}} \otimes_{\mathbb{Z}} \mathbb{C}^{\times}$is a maximal torus in ${ }^{L} G$, and the group algebra $\mathbb{C} \mathscr{X}$ is the representation algebra of ${ }^{L} A$.

The double cosets of $\mathscr{I}$ in $G$ are naturally parametrized by the semidirect product $W^{a}=W \ltimes \mathscr{X}$ of the Weyl group $W$ and $\mathscr{X}$. Let $s_{\alpha} \in W$ denote the reflection corresponding to the simple root $\alpha$, and let $s_{o} \in W^{a}$ be the affine reflection. A basis for the algebra $\mathscr{H}_{K}$ is given by the elements

$$
T_{w}=\text { characteristic function of } \mathscr{I} \widetilde{w} \mathscr{I} \text {, }
$$

where $w \in W$ and $\widetilde{w} \in K$ is a representative of $w$. The algebra $\mathscr{H}_{K}$ is generated by the elements $T_{s_{\alpha}}, \alpha \in \Pi$. Of course, we have $T_{w w^{\prime}}=T_{w} \cdot T_{w^{\prime}}$ if $\ell\left(w w^{\prime}\right)=\ell(w)+\ell\left(w^{\prime}\right)$ and $\left(T_{s_{\alpha}}+1\right)\left(T_{s_{\alpha}}-q\right)=0$. Here, $q$ is the order of the residue field.

Let $\mathscr{X}^{+}=\{x \in \mathscr{X} \mid \check{\alpha}(x) \leq 0 \forall \check{\alpha} \in \check{\Pi}\}$. For $x \in \mathscr{X}^{+}$and $\varpi$ a prime element in $\mathbb{F}$, set

$$
\theta_{x}=\text { characteristic function of } \mathscr{I} x(\varpi) \mathscr{I} .
$$

An element $L \in \mathscr{X}$ can be written as the difference $L=x-y$ with $x$ and $y$ in $\mathscr{X}^{+}$. Then the element

$$
\theta_{L}=\theta_{x} \theta_{y}^{-1}
$$

does not depend on the particular choice of $x$ and $y$ used in writing $L=x-y$. The algebra $\mathscr{A}$ is the subalgebra generated by the $\theta_{L}$ 's. Identify $\mathbb{C} \mathscr{X}$ with $\mathscr{A}$ via the isomorphism $L \mapsto \theta_{L}$ for $L \in \mathscr{X}$. Each $L$ is a character of ${ }^{L} A$, hence we can view $\theta_{L}$ as a regular function on the torus ${ }^{L} A$ and $\mathscr{A}$ as the algebra of regular functions on ${ }^{L} A$.

In order to describe the cross multiplication between the subalgebras $\mathscr{H}_{K}$ and $\mathscr{A}$, it is useful to recall [Ls2] the generic affine Hecke algebra $\mathscr{H}$ based on the datum $(\mathscr{X}, \mathscr{Y}, R, \check{R}, \Pi)$ with parameter set $c, c^{*}$. A parameter set is a pair of maps $c: \Pi \rightarrow \mathbb{N}$ and $c^{*}:\{\alpha \in \Pi, \check{\alpha} \in 2 \mathscr{Y}\} \rightarrow \mathbb{N}$ satisfying $c(\alpha)=c\left(\alpha^{\prime}\right)$ 
whenever $\left(\alpha, \check{\alpha}^{\prime}\right)=\left(\alpha^{\prime}, \check{\alpha}\right)=-1$. We write $c_{\alpha}, c_{\alpha}^{*}$ for the parameters. The special case of $\mathscr{H}(G / / \mathscr{I})$ in $(2.1)$ corresponds to all the $c_{\alpha}$ 's and the $c_{\alpha}^{*}$ 's being equal to 1 .

The generic affine algebra with parameters is an algebra $\mathscr{H}$ over $\mathbb{C}\left[z, z^{-1}\right]$, where $z$ is an indeterminant. It has generators $T_{\alpha}=T_{s_{\alpha}}(\alpha \in \Pi), \theta_{L}(L \in \mathscr{X})$ and relations

$$
\left(T_{\alpha}+1\right)\left(T_{\alpha}-z^{2 c(\alpha)}\right)=0 \quad \text { for } \alpha \in \Pi
$$

$$
T_{\alpha} T_{\alpha^{\prime}} T_{\alpha} \ldots=T_{\alpha^{\prime}} T_{\alpha} T_{\alpha^{\prime}} \ldots, \alpha \neq \alpha^{\prime}, m \text { factors on both sides, }
$$
where $m$ is the order of $s_{\alpha} s_{\alpha^{\prime}}$ in $W$;

where by Proposition 3.9 in [Ls2]

$$
\begin{gathered}
\theta_{L} \theta_{L^{\prime}}=\theta_{L+L^{\prime}}, \quad L, L^{\prime} \in \mathscr{X} ; \\
\theta_{L}=1 \text { when } L \text { is the trivial element of } \mathscr{X} ; \\
\theta_{L}\left(T_{s_{\alpha}}+1\right)-\left(T_{s_{\alpha}}+1\right) \theta_{s_{\alpha}(L)}=\left(\theta_{L}-\theta_{s_{\alpha}(L)}\right) \mathscr{G}_{\alpha},
\end{gathered}
$$

$$
\mathscr{G}_{\alpha}= \begin{cases}\frac{\theta_{\alpha} z^{2 c_{\alpha}}-1}{\theta_{\alpha}-1}, & \text { if } \check{\alpha} \notin 2 \mathscr{Y}, \\ \frac{\left(\theta_{\alpha} z^{c_{\alpha}+c_{\alpha}^{*}}-1\right)\left(\theta_{\alpha} z^{c_{\alpha}-c_{\alpha}^{*}}+1\right)}{\theta_{2 \alpha}-1} & \text { if } \check{\alpha} \in 2 \mathscr{Y} .\end{cases}
$$

Let $\mathscr{H}_{W}$ be the subalgebras of $\mathscr{H}$ generated by the $T_{\alpha}$ 's $(\alpha \in \Pi), \mathscr{A}$ the $\mathbb{C}$ subalgebra generated by the $\theta_{L}$ 's $(L \in \mathscr{X})$, and $\mathscr{A}\left[z, z^{-1}\right]=\mathbb{C}\left[z, z^{-1}\right] \otimes_{\mathbb{C}} \mathscr{A}$. As a $\mathbb{C}\left[z, z^{-1}\right]$-module, $\mathscr{H}$ has the decomposition

$$
\mathscr{H}=\mathscr{H}_{W} \underset{\mathbb{C}\left[z, z^{-1}\right]}{\otimes} \mathscr{A}\left[z, z^{-1}\right] .
$$

When the indeterminant $z$ is specialized to $q$, the order of the residue field, the algebras $\mathscr{H}, \mathscr{H}_{W}$, and $\mathscr{A}\left[z, z^{-1}\right]$ specialize to $\mathscr{H}(G / / \mathscr{I}), \mathscr{H}_{K}$, and $\mathscr{A}$ respectively.

We identify the algebra $\mathscr{A}\left[z, z^{-1}\right]$ with the algebra of regular functions on $\mathbb{C}^{\times} \times{ }^{L} A$. In this identification, the algebra $\mathscr{A}$ is the algebra of regular functions on ${ }^{L} A$, while $z$ and $z^{-1}$ generate the algebra of regular functions on $\mathbb{C}^{\times}$. The center of $\mathscr{H}$ is characterized as follows.

Theorem 2.1 (Bernstein). The center of $\mathscr{H}$ is $\mathscr{A}^{W} \otimes_{\mathbb{C}} \mathbb{C}\left[z, z^{-1}\right]$.

An immediate consequence of Theorem 2.1 is that a character $\chi$ of the center, also called an infinitesimal character, is given by a semisimple

$$
W \text {-orbit in } \mathbb{C}^{\times} \times{ }^{L} A \text {. }
$$

Let $\mathscr{O}$ be the corresponding orbit in ${ }^{L} A$ and $s$ a representative. The element $s$ has a canonical polar decomposition $s=s_{e} \cdot s_{h}$ into an elliptic and hyperbolic element. 
Definition 2.2. An infinitesimal character $\chi$ is called real if the corresponding $W$-orbit of $s_{e}$ consists of a single element.

The Weyl group $W$ acts as automorphisms on $\mathscr{A}\left[z, z^{-1}\right]$ by the formula $w\left(\theta_{L}\right)=\theta_{w(L)}$ and $w(z)=z$. The algebra $\mathscr{A}\left[z, z^{-1}\right]$ has no zero divisors. Let $\mathscr{F}$ be its quotient field. As in [Ls2], consider the two algebras defined as follows. The first algebra is

$$
\mathscr{H}(\mathscr{F})=\mathscr{H}_{W} \underset{\mathscr{A}\left[z, z^{-1}\right]}{\otimes} \mathscr{F},
$$

with cross multiplication between $\mathscr{H}_{W}$ and $\mathscr{F}$ given by the obvious extension of $(2.5 \mathrm{e})$. The second algebra is the algebra semidirect product

$$
\mathbb{C} W \ltimes \mathscr{F},
$$

with multiplication given by

$$
\left(w_{1} \otimes f_{1}\right)\left(w_{2} \otimes f_{2}\right)=w_{1} w_{2} \otimes w_{2}\left(f_{1}\right) f_{2} .
$$

An important result on the two algebras is the following.

Theorem 2.3 [Ls2, Proposition 5.2]. The map

$$
\begin{aligned}
& l: \mathbb{C} W \ltimes \mathscr{F} \rightarrow \mathscr{H}(\mathscr{F}), \\
& l\left(t_{\alpha}\right)=\left(T_{\alpha}+1\right) \mathscr{G}_{\alpha}^{-1}-1, \\
& l(f)=f \quad(f \in \mathscr{F})
\end{aligned}
$$

defines an algebra isomorphism.

\section{Graded algebras}

In [Ls2], Lusztig associates a graded Hecke algebra $\mathbb{H}$ to the Hecke algebra $\mathscr{H}$. Lusztig defines the graded Hecke algebra $\mathbb{H}$ as follows. Let $\mathscr{I}$ be the ideal in $\mathscr{A}\left[z, z^{-1}\right]$ consisting of the regular functions on $\mathbb{C}^{\times} \times{ }^{L} A$ which vanish at the element $(1,1)$. For $k$ a natural integer, $\tilde{I}^{k}=\mathscr{H} \cdot \mathscr{J}^{k}$ is an ideal in $\mathscr{H}$. The graded Hecke algebra $\mathbb{H}$ is obtained from $\mathscr{H}$ as the graded algebra with respect to the descending sequence of ideals $\tilde{\mathscr{I}}^{1} \supset \tilde{\mathscr{I}}^{2} \supset \cdots \supset \tilde{\mathscr{I}}^{k} \supset \cdots$. We need a generalization of Lusztig's construction. Observe that the single element $1 \in^{L} A$ is $W$-invariant. Our generalization consists of replacing 1 by a finite $W$-invariant set $\mathscr{O}$ in ${ }^{L} A$. In analogy with 1 , the set $\mathscr{O}$ defines an ideal $\mathscr{I}$ in $\mathscr{A}\left[z, z^{-1}\right]$, namely,

$$
\mathscr{I}=\left\{f: \mathbb{C}^{\times} \times{ }^{L} A \longrightarrow \mathbb{C} \mid f(1, \sigma)=0 \text { for all } \sigma \in \mathscr{O}\right\}
$$

The ideal $\mathscr{I}^{k}$ consists of those functions which vanish to order at least $k$ at every element in $\mathscr{O}$. Let $\tilde{I}^{k}=\mathscr{H} \cdot \mathscr{I}^{k}$. Exactly as in [Ls2], $\mathscr{H} \cdot \mathscr{I}^{k}=\mathscr{I}^{k} \cdot \mathscr{H}$ so that

$$
\tilde{\mathscr{I}}^{1} \supset \tilde{\mathscr{I}}^{2} \supset \cdots \supset \tilde{\mathscr{I}}^{k} \supset \cdots
$$

is a descending sequence of ideals. 
Definition 3.1. Let $\mathbb{H}_{\mathscr{O}}$ be the graded algebra obtained from $\mathscr{H}$ and the sequence of ideals in (3.2).

We summarize the properties of $\mathbb{H}_{\mathscr{Q}}$ relevant for our needs. The proofs are slight modifications of those found in [Ls2].

Define elements in $\mathbb{H}_{\mathscr{Q}}$ as follows:

$$
\begin{aligned}
r & =z-1 \quad(\bmod \tilde{\mathscr{I}}) \\
t_{\alpha} & =T_{s_{\alpha}} \quad(\bmod \tilde{\mathscr{I}}), \quad \alpha \in \Pi .
\end{aligned}
$$

The element $r$ is central in $\mathbb{H}_{\mathscr{O}}$ and by $(2.5 \mathrm{a}, \mathrm{b})$

(3.4) $t_{\alpha}^{2}=1$ and $\left(t_{\alpha} t_{\alpha^{\prime}}\right)^{m}=1 \quad$ where $m$ is the order of $s_{\alpha} s_{\alpha^{\prime}}$ in $W$.

The subalgebra generated by the $t_{\alpha}$ 's is canonically the group algebra $\mathbb{C W}$.

Let $\left\{F_{\sigma}\right\}_{\sigma \in \mathcal{O}}$ be functions on ${ }^{L} A$ satisfying

$$
F_{\sigma}-\delta_{\sigma, \sigma^{\prime}} \text { vanishes to order at least } 2 \text { at each } \sigma^{\prime} \in \mathscr{O} \text {. }
$$

In $\mathbb{H}_{\mathscr{O}}$, the elements

$$
E_{\sigma}=F_{\sigma}(\bmod \tilde{\mathscr{I}})
$$

are well defined and satisfy

$$
\sum_{\sigma \in \mathscr{O}} E_{\sigma}=1, \quad E_{\sigma} E_{\sigma^{\prime}}=\delta_{\sigma, \sigma^{\prime}} E_{\sigma} \text { and } E_{\sigma} t_{\alpha}=t_{\alpha} E_{s_{\alpha}(\sigma)} .
$$

For $\theta=\theta_{L} \in \mathscr{A}$ and $\sigma \in \mathscr{O}$, set

$$
\begin{aligned}
E_{\sigma} \omega_{\theta} & =\frac{\theta-\theta(\sigma)}{\theta(\sigma)} F_{\sigma} \quad\left(\bmod \tilde{\mathcal{I}}^{2}\right), \\
\omega_{\theta} & =\sum_{\sigma \in \mathscr{\theta}} E_{\sigma} \omega_{\theta} .
\end{aligned}
$$

Then

$$
\omega_{\theta_{1} \theta_{2}}=\omega_{\theta_{1}}+\omega_{\theta_{2}} \quad \text { for } \theta_{1}=\theta_{L_{1}}, \theta_{2}=\theta_{L_{2}} \in \mathscr{A}
$$

and

$$
\omega_{s(\theta)}=\omega_{\theta}-\langle L, \check{\alpha}\rangle \omega_{\alpha} \text { for } s=s_{\alpha} \in W .
$$

The Weyl group $W$ acts on the algebra spanned by the $\omega_{\theta}$ 's via (3.7b). This algebra is clearly canonically $W$-isomorphic to the symmetric algebra $\mathscr{S}$ of $\mathscr{L}=\mathscr{Z} \otimes_{\mathbb{Z}} \mathbb{C}$. Let $\mathscr{E}$ be the commutative $\mathbb{C}$-algebra generated by the $E_{\sigma}$ 's and $\mathbb{A}$ the commutative algebra $\mathscr{E} \otimes_{\mathbb{C}} \mathscr{S}$.

Proposition 3.2 [Ls2, Proposition 4.4]. (1) If $s=s_{\alpha}$, then

$$
\omega \cdot t_{s}-t_{s} \cdot s(\omega)=r\langle\omega, \check{\alpha}\rangle \sum_{\sigma \in \mathscr{O}} E_{\sigma} h_{\sigma, \alpha},
$$


where

$$
h_{\sigma, \alpha}= \begin{cases}0 & \text { if } s \sigma \neq \sigma, \\ 2 c_{\alpha} & \text { if } s \sigma=\sigma, \alpha \notin 2 \mathscr{Y}, \\ c_{\alpha}+c_{\alpha}^{*} \theta_{-\alpha}(\sigma) & \text { if } s \sigma=\sigma, \alpha \in 2 \mathscr{Y} .\end{cases}
$$

(2) The generator $r$ lies in the center of $\mathbb{H}_{\mathscr{O}}$ and

$$
\mathbb{H}_{\mathscr{O}}=\mathbb{C} W \otimes_{\mathbb{C}}\left(\mathbb{C}[r] \otimes_{\mathbb{C}} \mathbb{A}\right)
$$

with the cross multiplication between $\mathbb{C} W$ and $\mathbb{A}$ given by (3.8).

(3) If $\mathscr{O}$ is the disjoint union of two $W$-invariant sets $\mathscr{O}_{1}$ and $\mathscr{O}_{2}$, then as algebras $\mathbb{H}_{\mathscr{O}}=\mathbb{H}_{\mathscr{O}_{1}} \oplus \mathbb{H}_{\mathscr{O}_{2}}$.

(4) The center of $\mathbb{H}_{\mathscr{O}}$ is $\mathbb{C}[r] \otimes_{\mathbb{C}} \mathbb{A}^{W}$.

The description of $\mathbb{H}_{\mathscr{O}}$ given in Proposition 3.2 leads immedately to a description of the characters of the center. We can assume that $\mathscr{O}=W \cdot \sigma$ is a single orbit. Let $\operatorname{Stab}(\sigma)=\{w \in W \mid w(\sigma)=\sigma\}$. Multiplication by $E_{\sigma}$ defines an isomorphism between the Weyl group invariant elements of $\mathbb{A}$ and the $\operatorname{Stab}(\sigma)$ invariant elements in $\mathscr{S}$. Under this identification, an infinitesimal character for $\mathbb{H}_{\mathscr{O}}$ is a

$$
\operatorname{Stab}(\sigma) \text {-orbit in } \mathbb{C} \times\left(\check{\mathscr{X}} \otimes_{\mathbb{Z}} \mathbb{C}\right) .
$$

We recall some notation from [Ls2] describing the root datum necessary for defining a smaller graded Hecke algebra. Set:

$$
\begin{aligned}
& \operatorname{Stab}(\sigma)=\{w \in W \mid w(\sigma)=\sigma\} ; \\
& R_{\sigma}=\left\{\alpha \in R \mid \theta_{\alpha}(\sigma)= \begin{cases}1 & \text { if } \check{\alpha} \notin 2 \mathscr{Y}, \\
\pm 1 & \text { if } \check{\alpha} \in 2 \mathscr{Y} ;\end{cases} \right. \\
& R_{\sigma}^{+}=R_{\sigma} \cap R^{+} \text {; } \\
& \Pi_{\sigma}=\left\{\alpha \in R_{\sigma}^{+} \mid \alpha \text { is simple for } R_{\sigma}^{+}\right\} \text {; } \\
& W_{\sigma}=\text { subgroup of } W \text { generated by } s_{\alpha}\left(\alpha \in \Pi_{\sigma}\right) \text {; } \\
& \Gamma_{\sigma}=\left\{w \in \operatorname{Stab}(\sigma) \mid w\left(R_{\sigma}^{+}\right)=R_{\sigma}^{+}\right\} .
\end{aligned}
$$

Let $\mathscr{H}_{\sigma}$ be the Hecke algebra associated to the root system $\left(\mathscr{X}, \mathscr{Y}, R, \check{R}_{\sigma}\right.$, $\left.\Pi_{\sigma}\right)$. The Weyl group of this root system is $W_{\sigma}$. The element $\sigma$ is of course $W_{\sigma}$-invariant. Denote the graded version of $\mathscr{H}_{\sigma}{ }^{\sigma}$ at $\sigma$ as $\mathbb{H}_{\sigma}$. By (3.10),

$$
\mathbb{H}_{\sigma}=\mathbb{C} W_{\sigma} \otimes_{\mathbb{C}} \mathscr{S}[r],
$$

where $\mathscr{S}$ is the algebra of polynomial functions on $\check{\mathscr{X}} \otimes_{\mathbb{Z}} \mathbb{C}$. The group $\Gamma_{\sigma}$ acts as algebra automorphisms of $\mathbb{H}_{\sigma}$ via its actions on $W_{\sigma}$ and $\mathscr{S}$. Let

$$
\mathbb{H}_{\sigma}{ }^{\prime}=\Gamma_{\sigma} \ltimes \mathbb{H}_{\sigma}
$$

denote the algebra semidirect product.

Let $\left\{w_{1}=1, w_{2}, \ldots, w_{n}\right\}$ be coset representatives of $W / \operatorname{Stab}(\sigma)$. In particular, the elements of $\mathscr{O}$ are given by $\tau_{i}=w_{i} \sigma$. Define, as in [Ls2],

$$
E_{i, j}=t_{w_{i}^{-1}} E_{\sigma} t_{w_{j}}
$$

Clearly, $E_{\tau_{i}}=E_{i, i}$. 
Theorem 3.3. (1) For each $\tau=\tau_{i}, E_{\tau} \cdot \mathbb{H}_{\mathscr{O}} \cdot E_{\tau}$ is naturally isomorphic to $\mathscr{H}_{\sigma}^{\prime}$.

(2) The $E_{i, j}$ 's generate an $n \times n$ matrix algebra $\mathscr{M}_{n}$. The algebra $\mathbb{H}_{\mathscr{O}}$ is naturally isomorphic to the matrix algebra

$$
M_{n}\left(\mathbb{H}_{\sigma}^{\prime}\right)=\mathscr{M}_{n} \otimes_{\mathbb{C}} \mathbb{H}_{\sigma}{ }^{\prime}
$$

The vector space $\mathscr{C}_{n}=\sum_{i=1}^{n} \mathbb{C} E_{i, 1}$ is, up to isomorphism, the unique irreducible representation of $\mathscr{M}_{n}$. The next corollary follows easily from Theorem 3.3.

Corollary 3.4. (1) The functor $\mathscr{V} \stackrel{\mathscr{F}}{\longrightarrow} \mathscr{C}_{n} \otimes_{\mathbb{C}} \mathscr{V}$ is an equivalence between the categories of finite-dimensional modules of $\Gamma_{\sigma} \ltimes \mathbb{H}_{\sigma}$ and $\mathbb{H}_{\mathscr{O}}$.

(2) As a $\mathbb{C W}$ module, $\mathscr{F}(\mathscr{V})=\operatorname{Ind}_{W_{\sigma}}^{W} \mathscr{V}$.

Following [Ls2], we describe a completion of $\mathbb{H}_{\mathscr{O}}$ which will be useful later. For our purposes it is sufficient to work in the setting of holomorphic functions rather than formal power series.

The algebras $\mathbb{C}[r], \mathscr{S}$, and $\mathbb{C}[r] \otimes_{\mathbb{C}} \mathscr{S}$ consist of the polynomial functions on $\mathbb{C}, \check{\mathscr{X}} \otimes_{\mathbb{Z}} \mathbb{C}$, and $M=\mathbb{C} \times\left(\check{\mathscr{X}} \otimes_{\mathbb{Z}} \mathbb{C}\right)$ respectively. Let $\widehat{\mathbb{C}}[r], \hat{\mathscr{S}}$, and $\widehat{\mathbb{C}}[r] \otimes_{\mathbb{C}} \widehat{\mathscr{S}}$ denote the corresponding algebras of holomorphic functions, and let $\mathscr{K}$ and $\widehat{\mathscr{K}}$ be the fields of rational and meromorphic functions on $M$. Set $\widehat{\mathbb{A}}=\mathbb{A} \otimes_{\mathscr{S}} \widehat{\mathscr{S}}$. In analogy with (3.10) let

$$
\widehat{\mathbb{H}}_{\mathscr{O}}=\mathbb{C} W \otimes_{\mathbb{C}}\left(\widehat{\mathbb{C}}[r] \otimes_{\mathbb{C}} \widehat{\mathbb{A}}\right),
$$

and in analogy with (2.8) set

$$
\begin{aligned}
& \mathbb{H}_{\mathscr{O}}(\mathscr{K})=\mathbb{C} W \otimes\left(\mathscr{E} \otimes_{\mathbb{C}} \mathscr{K}\right) \supset \mathbb{H}_{\mathscr{O}}, \\
& \widehat{\mathbb{H}}_{\mathscr{O}}(\widehat{\mathscr{K}})=\mathbb{C} W \otimes\left(\mathscr{E} \otimes_{\mathbb{C}} \widehat{\mathscr{K}}\right) \supset \widehat{\mathbb{H}}_{\mathscr{O}} .
\end{aligned}
$$

Multiplication for these algebras are defined via (3.8). The algebra $\widehat{\mathbb{H}}_{\mathscr{O}}(\widehat{\mathscr{K}})$ contains $\mathbb{H}_{\mathscr{O}}(\mathscr{K})$. In [Ls2], Lusztig shows for $\mathscr{O}=\{1\}$ that the algebra $\widehat{\mathbb{H}}_{\mathscr{O}}(\widehat{\mathscr{K}})$ is naturally isomorphic to a semidirect product algebra (cf. Theorem 2.1). Lusztig's methods easily generalize to our setting, i.e., consider as in (2.8) the semidirect product algebra

$$
\mathbb{C} W \ltimes\left(\mathscr{E} \otimes_{\mathbb{C}} \widehat{\mathscr{K}}\right) .
$$

\section{Let}

$$
g_{\sigma, \alpha}=1+\frac{r h_{\sigma, \alpha}}{\alpha} .
$$

Theorem 3.5 [Ls2, Proposition 5.2]. The map

$$
\begin{aligned}
& l: \mathbb{C} W \ltimes\left(\mathscr{E} \otimes_{\mathbb{C}} \widehat{\mathscr{K}}\right) \longrightarrow \widehat{\mathbb{H}}_{\mathscr{O}}(\widehat{\mathscr{K}}), \\
& l\left(E_{\sigma}\right)=E_{\sigma}, \\
& l(f)=f \quad(f \in \mathscr{F}), \\
& l\left(t_{\alpha}\right)=\left(t_{\alpha}+1\right)\left(\sum_{\sigma} g_{\sigma, \alpha}^{-1} E_{\sigma}\right)-1
\end{aligned}
$$

defines an algebra isomorphism. 


\section{ANAlytic FAMilies}

Given a character $\chi$ of the center $\mathscr{Z}$ of $\mathscr{H}$, let $\mathscr{I}_{\chi}=\{z \in \mathscr{Z} \mid \chi(z)=0\}$ be the kernel of $\chi$. Both $\mathscr{A}\left[z, z^{-1}\right] \cdot \mathscr{I}_{\chi}$ and $\mathscr{H} \cdot \mathscr{I}_{\chi}$ are ideals in $\mathscr{A}\left[z, z^{-1}\right]$ and $\mathscr{H}$ respectively. Let

$$
\begin{aligned}
\mathscr{A}_{\chi} & =\mathscr{A}\left[z, z^{-1}\right] /\left\{\mathscr{A}\left[z, z^{-1}\right] \cdot \mathscr{I}_{\chi}\right\}, \\
\mathscr{H}_{\chi} & =\mathscr{H} /\left\{\mathscr{H} \cdot \mathscr{I}_{\chi}\right\}
\end{aligned}
$$

be the quotient algebras. The analogues for the graded algebras $\mathbb{A}, \mathbb{H}_{\mathscr{O}}, \widehat{\mathbb{A}}$, and $\widehat{\mathbb{H}}_{\mathscr{Q}}$ are clear. If $\bar{\chi}$ is an infinitesimal character of $\mathbb{H}_{\mathscr{O}}$, set

$$
\begin{aligned}
& \mathbb{A}_{\bar{\chi}}=\mathbb{A} /\left\{\mathbb{A} \cdot \mathscr{I}_{\bar{\chi}}\right\}=\widehat{\mathbb{A}} /\left\{\widehat{\mathbb{A}} \cdot \widehat{\mathscr{I}}_{\bar{\chi}}\right\}=\widehat{\mathbb{A}}_{\bar{\chi}}, \\
& \mathbb{H}_{\bar{\chi}}=\mathbb{H}_{\mathscr{O}} /\left\{\mathbb{H}_{\mathscr{O}} \cdot \mathscr{I}_{\bar{\chi}}\right\}=\widehat{\mathbb{H}}_{\mathscr{O}} /\left\{\widehat{\mathbb{H}}_{\mathscr{O}} \cdot \hat{\mathscr{I}}_{\bar{\chi}}\right\}=\widehat{\mathbb{H}}_{\bar{\chi}} .
\end{aligned}
$$

The main result in this section is Theorem 4.3. It gives conditions under which it is possible to match an infinitesimal character $\bar{\chi}$ of $\mathbb{H}_{\mathscr{Q}}$ to an infinitesimal character $\chi$ of $\mathscr{H}$ so that there are compatible natural isomorphisms from $\mathbb{A}_{\bar{\chi}}$ to $\mathscr{A}_{\chi}$ and from $\mathbb{H}_{\bar{\chi}}$ to $\mathscr{H}_{\chi}$. Theorem 4.3 is essentially Theorem 9.3 in [Ls2], the difference being that for applications to unitarity, we need to verify that the isomorphism is analytic in the $\nu$ parameter.

Assume $\mathscr{O}=W \cdot \sigma$ is the Weyl group orbit of an element $\sigma$ with trivial hyperbolic part. Let $\operatorname{Stab}(\sigma)$ be the centralizer of $\sigma$. By (2.7) and (3.11), infinitesimal characters for $\mathscr{H}$ and $\mathbb{H}_{\mathscr{Q}}$ are parameterized by $W$-orbits in $\mathbb{C}^{\times} \times$ ${ }^{L} A$ and $\operatorname{Stab}(\sigma)$-orbits in $\mathbb{C} \times\left(\check{\mathscr{X}} \otimes_{\mathbb{Z}} \mathbb{C}\right)$ respectively.

Proposition 4.1. (1) The map

$$
\begin{aligned}
& \mathbb{C} \times\left(\check{\mathscr{X}} \otimes_{\mathbb{Z}} \mathbb{C}\right) \rightarrow \mathbb{C}^{\times} \times{ }^{L} A, \\
& (r, t) \mapsto\left(e^{r}, \sigma \cdot e^{t}\right)
\end{aligned}
$$

is $\operatorname{Stab}(\sigma)$-equivariant. It matches the infinitesimal character $\chi=W\left(e^{r}, \sigma \cdot e^{t}\right)$ with the infinitesimal character $\bar{\chi}=\operatorname{Stab}(\sigma)(r, t)$. The map $\phi$ defined by

$$
\begin{aligned}
& \phi: \mathscr{A}\left[z, z^{-1}\right] \rightarrow \widehat{\mathbb{C}}[r] \otimes_{\mathbb{C}} \widehat{\mathbb{A}}, \\
& \phi(z)=e^{r}, \\
& \phi\left(\theta_{x}\right)=\sum_{\sigma \in \mathscr{O}} \theta_{x}(\sigma) \cdot E_{\sigma} \cdot e^{x} \quad(x \in \mathscr{X} \subset \mathscr{S})
\end{aligned}
$$

is a $\mathbb{C}$-algebra homomorphism. It maps $\mathscr{I}_{\chi}$ to $\mathscr{F}_{\bar{\chi}}$ and defines, by passage to the quotients, an isomorphism between $\mathscr{A}_{\chi}$ and $\mathbb{A}_{\bar{\chi}}$.

(2) The map

$$
\begin{aligned}
& \Phi: \mathscr{H} \longrightarrow \widehat{\mathbb{H}}_{\mathscr{O}}(\widehat{\mathscr{K}}), \\
& \Phi(a)=\phi(a) \quad\left(a \in \mathscr{A}\left[z, z^{-1}\right]\right), \\
& \Phi\left(T_{s}+1\right)=\sum_{\sigma \in \mathscr{O}} E_{\sigma}\left(t_{s}+1\right) \frac{\phi\left(\mathscr{G}_{\alpha}\right)}{g_{\sigma, \alpha}},
\end{aligned}
$$


where $\mathscr{G}_{\alpha}$ and $g_{\sigma, \alpha}$ are defined as in (2.5f) and (3.19) respectively, is an algebra homomorphism.

Proof. Clearly the map defined by (4.3) is $\operatorname{Stab}(\sigma)$-equivariant. It matches infinitesimal characters because if $\sigma \cdot e^{t}$ and $\sigma \cdot e^{t^{\prime}}$ represent $\chi$ then $t$ and $t^{\prime}$ must be conjugate by an element of $\operatorname{Stab}(\sigma)$. Consider now the map $\phi$. That $\phi$ is a $\mathbb{C}$-algebra homomorphism follows from the definition. Furthermore, if $y=\sum_{x} c_{x} \theta_{x}$, then

$$
\phi(y)=\sum_{x} c_{x} \sum_{\sigma \in \mathscr{O}} \theta_{x}(\sigma) E_{\sigma} e^{x}=\sum_{\sigma \in \mathscr{O}} E_{\sigma}\left(\sum_{x} c_{x} \theta_{x}(\sigma) e^{x}\right) .
$$

Relating the coefficient of $E_{\sigma}$ to the coefficient of $E_{w(\sigma)}$, we see that $\phi(y)$ lies in $\widehat{\mathbb{A}}^{W}$ if and only if $y$ is in $\mathscr{A}^{W}$. This means $\phi\left(\mathscr{F}_{\chi}\right) \subset \mathscr{F}_{\bar{\chi}}$, which in turn implies both the existence of the quotient map from $\mathscr{A}_{\chi}$ to $\mathbb{A}_{\bar{\chi}}$ and its injectivity. Since both $\mathscr{A}_{\chi}$ and $\mathbb{A}_{\bar{\chi}}$ are of dimension $|W|$ over $\mathbb{C}$, the homomorphism is in fact an isomorphism.

For part (2), note that $\Phi$ is the composition of $\left.\bar{l} \circ($ id, $\phi) \circ l^{-1}\right|_{\mathscr{H}}$, where $l$ is defined in Theorem 2.3, $\bar{l}$ is defined in Theorem 3.5, and

$$
\begin{aligned}
& (\text { id }, \phi): \mathbb{C} W \ltimes \mathscr{F} \rightarrow \mathbb{C} W \ltimes\left(\mathscr{E} \otimes_{\mathbb{C}} \widehat{\mathscr{K}}\right), \\
& (\text { id }, \phi):(w, f) \mapsto(w, \phi(f)) .
\end{aligned}
$$

That $\Phi$ is an isomorphism follows from the definitions of the cross multiplications in terms of $\mathscr{G}_{\alpha}$ and $g_{\sigma, \alpha}$.

Definition 4.2. Suppose $M$ is a real analytic manifold. An analytic family of $\mathbb{C}$-algebras depending on a parameter $\nu \in M$ is a finite-dimensional space $A$ equipped with an algebra multiplication ${ }_{\nu}$ such that, for each linear functional $\lambda \in \operatorname{Hom}_{\mathbb{C}}(A, \mathbb{C})$ and any $a_{1}, a_{2} \in A$,

$$
\lambda\left(a_{1} \cdot{ }_{\nu} a_{2}\right): M \rightarrow \mathbb{C}
$$

is real analytic. In coordinates this means that if we choose a basis $\left\{x_{1}, \ldots, x_{m}\right\}$ then

$$
x_{i} \cdot{ }_{\nu} x_{j}=\sum_{k} c_{i j}^{k}(\nu) x_{k}
$$

where the $c_{i j}^{k}$ are real analytic for $\nu \in M$.

A morphism of analytic families is an algebra map $\Phi_{\nu}: A \rightarrow B$ such that when $\Phi_{\nu}$ is expressed as a matrix with respect to the basis $\left\{x_{1}, \ldots, x_{m}\right\}$, all entries are analytic in $\nu$. Clearly these notions are independent of the basis chosen.

The algebras $\mathscr{A}\left[z, z^{-1}\right]$ and $\widehat{\mathbb{A}} \otimes_{\mathbb{C}} \widehat{\mathbb{C}}[r]$ can be used to construct analytic families of $\mathbb{C}$-algebras of dimension $|W|$ parameterized by $M=\mathbb{C} \times\left(\check{\mathscr{X}}_{\mathbb{Z}} \mathbb{C}\right)$ which contain $\mathscr{A}_{\chi}$ and $\mathbb{A} \bar{\chi}=\widehat{\mathbb{A}} \bar{\chi}$ respectively. To see this, we choose a basis $\left\{a_{1}, \ldots, a_{m}\right\}(m=|W|)$ of $\mathscr{A}$ as a free $\mathscr{A}^{W}$ module. Define $c_{i, j}^{k} \in \mathscr{A}^{W}$ by

$$
a_{i} \cdot a_{j}=\sum_{k} c_{i, j}^{k} a_{k}
$$


By Proposition 4.1(1), $\phi\left(a_{i}\right)$ is a basis of $\widehat{\mathbb{A}}$ as a free $\widehat{\mathbb{A}}^{W}$ module.

For $\nu=(r, t) \in M$ and $\chi=W\left(e^{r}, \sigma \cdot e^{t}\right)$, the algebra $A_{\chi}$ is just the finite-dimensional algebra $A_{\nu}$ given formally as the commutative algebra with $\mathbb{C}$-basis $a_{i}$ and multiplication

$$
a_{i}{ }_{\nu} a_{j}=\sum_{k} c_{i, j}^{k}(\chi) a_{k} .
$$

Similarly, $\mathbb{A} \bar{\chi}=\widehat{\mathbb{A}} \bar{\chi}$ is the commutative algebra $\widehat{\mathbb{A}}_{\nu}$ with formal $\mathbb{C}$-basis $\phi\left(a_{i}\right)$ and multiplication

$$
\phi\left(a_{i}\right) \cdot{ }_{\nu} \phi\left(a_{j}\right)=\sum_{k} \phi\left(c_{i, j}^{k}\right)(\bar{\chi}) \phi\left(a_{k}\right) .
$$

In terms of analytic families, since $c_{i, j}^{k}(\chi)=\phi\left(c_{i, j}^{k}\right)(\bar{\chi})$, the map $a_{i} \mapsto \phi\left(a_{i}\right)$ on the basis vectors is an isomorphism between $A_{\nu}$ and $\widehat{\mathbb{A}}_{\nu}$.

In a similar fashion, the three algebras $\mathscr{H}, \mathbb{H}_{\mathscr{O}}$, and $\widehat{\mathbb{H}}_{\mathscr{O}}$ can also be viewed as analytic families of $\mathbb{C}$-algebras of dimension $|W|^{2}$, i.e., used to construct $\mathbb{C}$-algebras of dimension $|W|^{2}$. The parameter space is the same. Inclusion of $\mathbb{H}_{\mathscr{O}}$ into $\widehat{\mathbb{H}}_{\mathscr{O}}$ yields an inclusion of analytic families. The main result we want to show is that $\mathscr{H}_{\chi}$ and $\mathbb{H}_{\bar{\chi}}$ are isomorphic under suitable conditions on the parameter $\nu$. We may of course view the three algebras as analytic families over $M_{\mathbb{R}}=\mathbb{R} \times\left(\check{\mathscr{X}} \otimes_{\mathbb{Z}} \mathbb{R}^{\times}\right) \subset M$.

Theorem 4.3. For $\nu \in M_{\mathbb{R}}$ the homomorphism $\Phi_{\nu}: \mathscr{H}_{\chi} \rightarrow \widehat{\mathbb{H}}_{\bar{\chi}}$ defined via Proposition 4.2 is an analytic isomorphism of the two families of algebras.

Proof. It is enough to show $f_{\sigma, \alpha}=\phi\left(\mathscr{G}_{\alpha}\right) / g_{\sigma, \alpha} \in \widehat{\mathscr{K}}$ is analytic and nowhere zero on $M_{\mathbb{R}}$. Adapting the proof of Lemma 9.5 in [Ls2] to our situation, we find

$$
f_{\sigma, \alpha}= \begin{cases}\frac{e^{2 r c_{\alpha}} e^{\alpha} \theta_{\alpha}(\sigma)-1}{e^{\alpha} \theta_{\alpha}(\sigma)-1} & \text { if } s \sigma \neq \sigma, \\ \frac{e^{2 r c_{\alpha}} e^{\alpha}-1}{e^{\alpha}-1} \cdot \frac{\alpha}{2 r c_{\alpha}+\alpha} & \text { if } s \sigma=\sigma, \check{\alpha} \notin 2 \mathscr{Y}, \\ \frac{\left(e^{r\left(c_{\alpha}+c_{\alpha}^{*}\right)} e^{\alpha} \theta_{\alpha}(\sigma)-1\right)\left(e^{r\left(c_{\alpha}-c_{\alpha}^{*}\right)} e^{\alpha} \theta_{\alpha}(\sigma)+1\right) \alpha}{\left(e^{2 \alpha}-1\right)\left\{r\left(c_{\alpha}+c_{\alpha}^{*} \theta_{-\alpha}(\sigma)\right)+\alpha\right\}} & \text { if } s \sigma=\sigma, \check{\alpha} \in 2 \mathscr{Y} .\end{cases}
$$

Note that $\theta_{\alpha}(\sigma)$ is not a positive real number if $s(\sigma) \neq \sigma$. Also, if $s(\sigma)=\sigma$ then

$$
\theta_{\alpha}(\sigma)= \begin{cases}1 & \text { if } \check{\alpha} \notin 2 \mathscr{Y}, \\ \pm 1 & \text { if } \check{\alpha} \in 2 \mathscr{Y} .\end{cases}
$$

It is clear that $f_{\sigma, \alpha}$ is a nonzero nonvanishing function on $M_{\mathbb{R}}$. In particular, $\Phi_{\nu}$ is an isomorphism for all $\bar{\chi}=W_{\sigma}$-orbit of $(r, \nu)$.

\section{Hermitian structures}

An important property of $\mathscr{H}(G / / \mathscr{I})$ is that it is a $*$-algebra with an inner product. For $f_{1}, f_{2} \in \mathscr{H}(G / / \mathscr{I})$, 


$$
\begin{aligned}
f_{i}^{*}(g) & =\overline{f_{i}\left(g^{-1}\right)}, \\
\left\langle f_{1}, f_{2}\right\rangle & =\int_{G} f_{1}(g) \overline{f_{2}(g)} d g=f_{1} * f_{2}^{*}(1) .
\end{aligned}
$$

There is a natural ${ }^{*}$ operation on the generic affine Hecke algebra $\mathscr{H}$ compatible with the corresponding one on $\mathscr{H}(G / / \mathscr{I})$. We describe ${ }^{*}$ on a set of generators. Assume $R$ is irreducible. Recall that $\mathscr{H}$ is generated by the elements

$$
\left\{T_{\alpha}, T_{o}, \tau, \theta_{x}\right\}_{\alpha \in \Pi, x \in \mathscr{L}_{0}},
$$

where the $T_{\alpha}$ 's and $T_{0}$ are generators indexed by the simple reflections in the affine Weyl group and

$$
\mathscr{X}_{0}=\{x \in \mathscr{X} \mid\langle x, \check{\alpha}\rangle=0 \text { for all } \check{\alpha} \in \check{R}\} .
$$

We need to describe $\tau$. The group $A_{s}=\left\{x(a) \in G \mid x \in \mathscr{X}_{0}, a \in \mathbb{F}\right\}$ is the split component of the center of $G$. Let $N_{s}$ be the normalizer of $A_{s} \mathscr{I}$ in $G$. Denote by $\mathscr{Q}$ the $\mathbb{Z}$ span of the elements in $R$. By [S], we have $\mathscr{Z}_{0} \cap \mathscr{Q}=$ $\varnothing$ and $\mathscr{X} /\left[\mathscr{Q}+\mathscr{X}_{0}\right]$ is finite and isomorphic to $N_{s} /\left\{A_{s} \mathscr{I}\right\}$. Because $R$ is irreducible, these quotients are cyclic. Let $t A_{s} \mathscr{I}$ be a generator of $N_{s} /\left\{A_{s} \mathscr{I}\right\}$. Then $\mathscr{I} t=t \mathscr{I}=\mathscr{I} t \mathscr{I}$. In $\mathscr{H}(G / / \mathscr{I})$, the characteristic function $T_{t}$ of $\mathscr{I} t \mathscr{I}$ is invertible. The inverse is the characteristic function of $\mathscr{I}_{t}^{-1} \mathscr{I}=$ $\mathscr{I} t^{-1}=t^{-1} \mathscr{I}$. Let $\tau$ be the element in $\mathscr{H}$ which goes to $T_{t}$. In terms of the generators $(5.2),{ }^{*}$ is the unique complex conjugate linear antiautomorphism which satisfies

$$
\begin{aligned}
z^{*} & =z, \\
T_{\alpha}^{*} & =T_{\alpha}, \\
T_{o}^{*} & =T_{o}, \\
\tau^{*} & =\tau^{-1}, \\
\theta_{x}^{*} & =\theta_{-x}=\theta_{x}^{-1}, \quad x \in \mathscr{X}_{0} \text { (split reductive group) } .
\end{aligned}
$$

The next lemma gives some indication of how $*$ behaves on $\mathscr{A}$.

\section{Lemma 5.1.}

$$
\theta^{*}=\theta^{-1}\left(\bmod \left(z-z^{-1}\right) \mathscr{H}\right)
$$

Proof. Observe that

$$
\begin{aligned}
T_{w}^{-1} & \equiv T_{w^{-1}} \quad\left(\bmod \left(z-z^{-1}\right) \mathscr{H}\right), \\
T_{w} \theta_{x} & \equiv \theta_{w(x)} T_{w} \quad\left(\bmod \left(z-z^{-1}\right) \mathscr{H}\right) .
\end{aligned}
$$

In view of the relations in (5.4) it is sufficient to prove (5.5) for one $0 \neq x \in \mathscr{Q}$. Let $\gamma$ be the negative of the highest short root in $R^{+}$and $T_{\gamma}=T_{s_{\gamma}}$ the element in $\mathscr{H}$ corresponding to the reflection $s_{\gamma} \in W$ about $\gamma$. Then

$$
\theta_{\gamma}=T_{o} T_{\gamma} \text {. }
$$


Choose a simple root $\alpha$ and a sequence of simple reflections $s_{1}, \ldots, s_{k}$ so that

$$
\gamma=s_{1} \cdots s_{k}(\alpha)
$$

In the Hecke algebra

$$
T_{\gamma}=T_{1} \cdots T_{k} \cdot T_{\alpha} \cdot T_{k} \cdots T_{1}
$$

Thus

$$
\theta_{\gamma}^{*}=\left(T_{\gamma}\right)^{*} T_{0}=T_{\gamma} \theta_{\gamma}\left(T_{\gamma}\right)^{-1}
$$

By (5.6) and (5.7), this gives

$$
\left(\theta_{\gamma}\right)^{*} \equiv\left(\theta_{\gamma}\right)^{-1}=\theta_{-\gamma} \quad\left(\bmod \left(z-z^{-1}\right) \mathscr{H}\right) .
$$

If $(\pi, V)$ is a finite-dimensional representation of $\mathscr{H}(G / / \mathscr{I})$, let $\left(\pi^{*}, V^{*}\right)$ be the hermitian dual with respect to ${ }^{*}$. To describe the hermitian dual of an irreducible representation, we review their classification. Kazhdan and Lusztig have shown in [KL] that the equivalence classes of irreducible modules for $\mathscr{H}$ are in 1-1 correspondence with ${ }^{L} G$ conjugacy classes of pairs $\{\phi, \rho\}$, where $\phi: \mathbb{Z} \times \operatorname{SL}(2, \mathbb{C}) \rightarrow{ }^{L} G$ is an admissible homomorphism, i.e., $\phi(1,1)$ is semisimple, and $\rho$ are certain characters of the component group of the centralizer of $\phi$. It is well known that $\phi$ is equivalent to a pair of elements $s, u \in{ }^{L} G$ with $s$ semisimple, $u$ unipotent, and $s u s^{-1}=u^{q}$. If $s \in{ }^{L} G$, let $s=s_{e} s_{h}$ be the polar decomposition of $s$ into its elliptic and hyperbolic parts. Set

$$
\bar{s}=s_{e} s_{h}^{-1} .
$$

For $n \in \mathbb{Z}$ and $g \in \operatorname{SL}(2, \mathbb{C})$, set $\bar{\phi}(n, g)=\overline{\phi(n, 1)} \phi(0, g)$.

Theorem 5.2. Let $(\pi, V)$ correspond to $\{\phi, \rho\}$. Then the hermitian dual $\left(\pi^{*}, V^{*}\right)$ corresponds to $\{\bar{\phi}, \rho\}$. In terms of the pair $\{s, u\}$ an irreducible module admits a hermitian structure precisely when $\bar{s}$ is ${ }^{L} G$ conjugate to $s$.

The proof is similar to the corresponding result for real reductive groups. We omit the details.

To proceed further, we need to understand when $\mathbb{H}_{\mathscr{Q}}$ is a $*$-algebra. If $s \in{ }^{L} A$, let $\bar{s}=s_{e} \cdot s_{h}^{-1}$ be as in (5.8) and set

$$
\mathscr{O}^{*}=\{\bar{\sigma} \mid \sigma \in \mathscr{O}\} \text {. }
$$

By Lemma 5.1 , the ideal of $\mathscr{H}$ corresponding to $\mathscr{O}^{*}$ is the $*$ of $\mathscr{H} \cdot \mathscr{I}$. The * operation on $\mathscr{H}$ induces an anti-isomorphism between $\mathbb{H}_{\mathscr{O}}$ and $\mathbb{H}_{\mathscr{O}^{*}}$. The * operation satisfies

$$
\begin{aligned}
r^{*} & =r, \\
w^{*} & =w^{-1} \quad(w \in W) .
\end{aligned}
$$

It follows from the relation

$$
\theta(\bar{\sigma})=\overline{\theta\left(\sigma^{-1}\right)}
$$


that

$$
E_{\sigma}^{*}=E_{\bar{\sigma}}
$$

Definition 5.3. The set $\mathscr{O}$ is said to be hermitian if it is equal to $\mathscr{O}^{*}$.

In this setting, it follows from Lemma 5.1 that the ideal $\mathscr{H} \cdot \mathscr{J}$ is $*$ invariant, so that $\mathbb{H}_{\mathscr{Q}}$ and $\widehat{\mathbb{H}}_{\mathscr{O}}$ are $*$ algebras. A particularly important instance in which $\mathscr{O}$ is hermitian is when it is the Weyl group orbit of an elliptic element. We assume for the remainder of the section that $\mathscr{O}$ is hermitian.

We compute a formula for $\omega^{*}$ for $\omega \in \mathbb{C} \otimes_{\mathbb{Z}} \mathscr{X}$.

Lemma 5.4. Let $\gamma \in R^{+}$be the highest short coroot, let $s_{\gamma} \in W$ be the corresponding reflection, and let $T_{\gamma}=T_{s_{\gamma}}$ be the corresponding element in $\mathscr{H}_{W}$. Then

$$
\gamma^{*}=t_{\gamma} \cdot \gamma \cdot t_{\gamma}=t_{l} \cdot \gamma \cdot t_{l}
$$

where $t_{l}$ is the element in the graded Hecke algebra corresponding to the long element $w_{l}$ in the Weyl group.

Proof. As in the proof of Lemma 5.1, choose a simple root $\alpha$ and a sequence of simple reflections $s_{1}, \ldots, s_{k}$ so that

$$
\gamma=s_{1} \cdots s_{k}(\alpha) \text {. }
$$

Then in the Hecke algebra

$$
T_{\gamma}=T_{1} \cdots T_{k} \cdot T_{\alpha} \cdot T_{k} \cdots T_{1},
$$

and similarly in the Weyl group. By relation (2.5a),

$$
T_{\beta}^{-1}=z^{-2 c(\beta)} T_{\beta}-z^{-2 c(\beta)}\left(z^{2 c(\beta)}-1\right) \equiv t_{\beta} \quad\left(\bmod \tilde{\mathcal{J}}^{*}\right) .
$$

Thus

$$
\left(T_{\gamma}\right)^{-1} \equiv t_{\gamma} \quad\left(\bmod \tilde{\mathscr{J}}^{*}\right)
$$

then

$$
\theta_{\gamma}=T_{0} \cdot T_{\gamma} \in \mathscr{X}^{+}
$$

To simplify notation, set $\theta=\theta_{\gamma}$. If we apply ${ }^{*}$ to (5.17) and use the fact that by $(5.14)\left(T_{\gamma}\right)^{*}=T_{\gamma}$, we get

$$
\theta^{*}=\left(T_{\gamma}\right)^{*} T_{0}=\left(T_{\gamma}\right)^{*} \theta T_{\gamma}^{-1}=T_{\gamma} \theta T_{\gamma}^{-1}
$$

Then

$$
\left(\sum_{\sigma \in \mathscr{O}} F_{\sigma} \frac{\theta-\theta(\sigma)}{\theta(\sigma)}\right)^{*}=\sum_{\sigma \in \mathscr{O}} \frac{T_{\gamma} \theta T_{\gamma}-\overline{\theta(\sigma)}}{\overline{\theta(\sigma)}}\left(F_{\sigma}\right)^{*}
$$


This expression is in $\tilde{\mathscr{J}}^{*}$ and we must grade it modulo $\left(\tilde{\mathscr{I}}^{*}\right)^{2}$. Thus we can take $\left(T_{\gamma}\right)^{*},\left(F_{\sigma}\right)^{*}$, and $\left(T_{\gamma}\right)^{-1}$ modulo $\tilde{\mathscr{J}}^{*}$. We get

$$
\begin{aligned}
\gamma^{*} & =\sum_{\sigma \in \mathscr{O}} \frac{t_{\gamma} \theta t_{\gamma}-\overline{\theta(\sigma)}}{\overline{\theta(\sigma)}} E_{\bar{\sigma}}\left(\bmod \tilde{\mathscr{I}}^{2}\right)=t_{\gamma} \sum_{\sigma \in \mathscr{O}} E_{\bar{\sigma}} \frac{\theta-\overline{\theta\left(t_{\gamma}(\sigma)\right)}}{\overline{\theta\left(t_{\gamma}(\sigma)\right)}} t_{\gamma} \\
& =t_{\gamma} \sum_{\sigma \in \mathscr{O}} E_{\bar{\sigma}} \frac{\theta-\theta(\bar{\sigma})}{\theta(\bar{\sigma})} t_{\gamma}=t_{\gamma} \gamma t_{\gamma} .
\end{aligned}
$$

The second part of (5.12) follows from the fact that $t_{l}=t_{\gamma} \cdot t^{\gamma}$, where $t^{\gamma}$ is a product of simple roots all commuting with $\gamma$.

We obtain a more general formula for ${ }^{*}$ on $\mathscr{S}$ as follows. For $\omega \in \mathscr{X}$, set

$$
\widetilde{\omega}=-t_{l}(\omega)
$$

and extend it to a conjugate linear automorphism of $\mathscr{S}$. This map defines a permutation of the set of simple roots. The map $\sim$ extends to an automorphism of $\mathscr{S}$.

Corollary 5.5. For $f \in \mathscr{S} \subset \mathbb{H}_{\mathscr{O}}$,

$$
f^{*}=t_{l} \cdot \tilde{f} \cdot t_{l}
$$

Proof. It is enough to prove the formula for $w(\gamma)$ with $w \in W$, or equivalently, it is enough to show that for $\alpha \in \Pi$ and $\omega \in \mathscr{X}$,

$$
\text { if } \omega^{*}=t_{l} \cdot \widetilde{\omega} \cdot t_{l} \text {, then } s_{\alpha}(\omega)^{*}=t_{l} \cdot \widetilde{s_{\alpha}(\omega)} \cdot t_{l} .
$$

By Proposition 3.2,

$$
s_{\alpha} \omega=t_{\alpha} \cdot \omega \cdot t_{\alpha}-r\langle\omega, \check{\alpha}\rangle \sum_{\sigma \in \mathscr{O}} E_{\sigma} h_{\sigma, \alpha} t_{\alpha} .
$$

Therefore,

$$
\begin{aligned}
s_{\alpha}(\omega)^{*} & =t_{\alpha} \cdot \omega^{*} \cdot t_{\alpha}-r\langle\omega, \check{\alpha}\rangle \sum_{\sigma \in \mathscr{O}} t_{\alpha} h_{\sigma, \alpha}^{*} E_{\sigma}^{*} \\
& =t_{\alpha} \cdot t_{l} \cdot \widetilde{\omega} \cdot t_{l} \cdot t_{\alpha}-r\langle\omega, \check{\alpha}\rangle \sum_{\sigma \in \mathscr{O}} t_{\alpha} h_{\sigma^{*}, \alpha} E_{\sigma^{*}} \\
& =t_{l} \cdot\left(t_{\widetilde{\alpha}} \cdot \widetilde{\omega} \cdot t_{\widetilde{\alpha}}\right) \cdot t_{l}-r\langle\omega, \check{\alpha}\rangle \sum_{\sigma \in \mathscr{O}} t_{\alpha} h_{\sigma^{*}, \alpha} E_{\sigma^{*}}
\end{aligned}
$$

Thus, by using the $*$-invariance of $\mathscr{O}$, we can rewrite this as

$$
\begin{aligned}
s_{\alpha}(\omega)^{*} & \left.=t_{l} \cdot\left[t_{\widetilde{\alpha}} \cdot \widetilde{\omega} \cdot t_{\widetilde{\alpha}}-r \sum_{\sigma \in \mathscr{O}} \widetilde{s_{\alpha}(\omega)}, \alpha\right\rangle t_{\widetilde{\alpha}} h_{\sigma, \alpha} E_{\sigma}\right] \cdot t_{l} \\
& =t_{l} \cdot s_{\widetilde{\alpha}}(\widetilde{\omega}) \cdot t_{l}=t_{l} \cdot \widetilde{s_{\alpha}(\omega)} \cdot t_{l} . \quad \square
\end{aligned}
$$

We now prove a different more explicit formula for *. Recall the definition of $h_{\sigma, \alpha}$ in Proposition 3.2. We extend it to any root $\beta \in R$, subject to $h_{w \sigma, w \alpha}=h_{\sigma, \alpha}$. This is well defined in view of the properties of the functions $c, c^{*}$. 
Theorem 5.6. For $\omega \in \mathscr{X}$,

$$
\omega^{*}=-\omega+r \sum_{\beta \in R^{+}} t_{\beta}\langle\omega, \check{\beta}\rangle \sum_{\sigma \in \mathscr{O}} E_{\sigma} h_{\sigma, \beta} .
$$

Proof. For each $\omega$, consider the expression

$$
\tau(\omega)=\omega-\frac{r}{2} \sum_{\beta \in R^{+}} t_{\beta}\langle\omega, \check{\beta}\rangle \sum_{\sigma \in \mathscr{O}} E_{\sigma} h_{\sigma, \beta} .
$$

We claim that $\tau(\omega)$ has the property that

$$
t_{w} \tau(\omega)=\tau(w(\omega)) t_{w} .
$$

To prove this it is enough to consider the case $w=s_{\alpha}$ for $\alpha$ a simple root. Here, it is enough to show that

$$
t_{s} \tau(\omega) t_{s}=\tau(s(\omega)) .
$$

By Proposition 3.2(1)

$$
t_{s} \omega t_{s}=s(\omega)+r\langle\omega, \check{\alpha}\rangle \sum_{\sigma \in \mathscr{O}} E_{\sigma} h_{\sigma, \alpha} t_{s} .
$$

On the other hand,

$$
\begin{aligned}
& t_{s} \sum_{\beta \in R^{+}} t_{\beta}\langle\omega, \check{\beta}\rangle \sum_{\sigma \in \mathscr{O}} E_{\sigma} h_{\sigma, \beta} \\
& =\sum_{\beta \in R^{+}} t_{s(\beta)}\langle\omega, \check{\beta}\rangle \sum_{\sigma \in \mathscr{O}} E_{s(\sigma)} h_{\sigma, \beta} t_{s} \\
& =\sum_{\beta \in R^{+} \backslash \alpha} t_{s(\beta)}\langle\omega, \check{\beta}\rangle \sum_{\sigma \in \mathscr{O}} E_{s(\sigma)} h_{\sigma, \beta} t_{s}+\langle\omega, \check{\alpha}\rangle \sum_{\sigma \in \mathscr{O}} E_{\sigma} h_{\sigma, \alpha} \\
& =\sum_{\beta \in R^{+}} t_{\beta}\langle\omega, \check{\beta}\rangle \sum_{\sigma \in \mathscr{O}} E_{\sigma} h_{\sigma, \beta} t_{s}-2 r\langle\omega, \check{\alpha}\rangle \sum_{\sigma \in \mathscr{O}} E_{\sigma} h_{\sigma, \alpha}
\end{aligned}
$$

because of (5.24). Formula (5.29) follows by combining (5.30) and (5.31).

We now apply this to the highest coroot $\gamma$. In view of Lemma 5.4,

$$
t_{\gamma}(\tau(\gamma)) t_{\gamma}=\tau\left(t_{\gamma}(\gamma)\right)=-\tau(\gamma)
$$

Therefore,

$$
\begin{aligned}
\gamma^{*}= & -\gamma+\frac{r}{2} t_{\gamma} \sum_{\beta \in R^{+}} t_{\beta}\langle\gamma, \check{\beta}\rangle \sum_{\sigma \in \mathscr{O}} E_{\sigma} h_{\sigma, \beta} t_{\gamma} \\
& +\frac{r}{2} \sum_{\beta \in R^{+}} t_{\beta}\langle\gamma, \check{\beta}\rangle \sum_{\sigma \in \mathscr{O}} E_{\sigma} h_{\sigma, \beta} .
\end{aligned}
$$

The proof is complete once we observe that

$$
t_{\gamma} \sum_{\beta \in R^{+}} t_{\beta}\langle\omega, \check{\beta}\rangle \sum_{\sigma \in \mathscr{O}} E_{\sigma} h_{\sigma, \beta} t_{\gamma}=\sum_{\beta \in R^{+}} t_{\beta}\langle\omega, \check{\beta}\rangle \sum_{\sigma \in \mathscr{O}} E_{\sigma} h_{\sigma, \beta} .
$$


This completes the proof of (5.27) for $\gamma$. Formula (5.27) now follows for any $w(\gamma)$ with $w \in W$ by

$$
\tau(w(\gamma))^{*}=\left(t_{w} \tau(\gamma) t_{w}^{-1}\right)^{*}=t_{w} \tau(\gamma)^{*} t_{w}^{-1}=-\tau(w(\gamma)) .
$$

The general case follows from the fact that $*$ is a (complex conjugate) antiautomorphism.

At this point we are ready to explain the relationship between the ${ }^{*}$ operation on $\mathscr{H}$ and the ${ }^{*}$ operation on $\mathbb{H}_{\mathscr{O}}$. Recall that $f_{\sigma, \alpha}=\phi\left(\mathscr{G}_{\alpha}\right) / g_{\sigma, \alpha}$. Define

$$
\begin{gathered}
F_{\sigma, \rho}=\prod_{\alpha \in R^{+}} f_{\sigma, \alpha}, \quad F_{\rho}=\sum E_{\sigma} F_{\sigma, \rho}, \\
m=\Phi\left(T_{l}\right) \cdot F_{\rho}^{-1} \cdot t_{l}
\end{gathered}
$$

where $T_{l}$ is the element in $\mathscr{H}$ corresponding to $w_{l}$.

Theorem 5.7. For $\nu$ real, the isomorphism $\Phi$ of Theorem 4.3 is compatible with the ${ }^{*}$ structures on $\mathscr{H}_{\chi}$ and $\mathbb{H}_{\bar{\chi}}$ in the following sense:

$$
\begin{aligned}
& \boldsymbol{\Phi}\left(T_{\alpha}^{*}\right)=m \cdot \Phi\left(T_{\alpha}\right)^{*} \cdot m^{-1}, \quad \alpha \in \Pi, \\
& \Phi\left(\theta^{*}\right)=m \cdot \Phi(\theta)^{*} \cdot m^{-1}, \quad \theta \in \mathscr{A} .
\end{aligned}
$$

Proof. The fact that $m$ is invertible follows from the fact that $f_{\sigma, \alpha}$ are analytic nowhere zero on $M_{\mathbb{R}}$ established in the proof of Theorem 4.3.

Observe first that $T_{\alpha}^{*}=T_{\alpha}$. Furthermore,

$$
\Phi\left(T_{\alpha}\right)^{*}=\left[t_{l} \tilde{f}_{\widetilde{\alpha}} t_{l}\left(t_{\alpha}+1\right)-1\right]=\left[f_{\widetilde{\alpha}}\left(t_{\widetilde{\alpha}}+1\right)-1\right] t_{l},
$$

and since $f_{\widetilde{\alpha}} F_{\rho}^{-1}$ is $t_{\widetilde{\alpha}}$ invariant,

$$
F_{\rho}^{-1}\left[\left[f_{\widetilde{\alpha}}\left(t_{\widetilde{\alpha}}+1\right)-1\right]=\left[f_{\widetilde{\alpha}}\left(t_{\widetilde{\alpha}}+1\right)-1\right] F_{\rho}^{-1}\right. \text {. }
$$

Then (5.37a) follows by applying (5.38) and (5.39).

For $(5.37 \mathrm{~b})$ it is enough to show the relation for $\theta=\theta_{\gamma}$. This is clear from Lemma 5.4 and Corollary 5.5.

Corollary 5.8. The relation

$$
m^{*}=m
$$

holds.

Proof. Relation (5.40) can be rewritten as

$$
\Phi\left(T_{l}\right)^{*}=t_{l} \cdot F_{\rho}^{-1} \cdot \Phi\left(T_{l}\right) \cdot F_{\rho} \cdot t_{l}
$$

because $\widetilde{F}_{\rho}=F_{\rho}$. Then $(5.41)$ follows by repeated application of $(5.37 \mathrm{a})$ and formulas (5.38) and (5.39).

Suppose $(\pi, V)$ is a representation of $\mathbb{H}_{\mathscr{O}}$ with infinitesimal character $\bar{\chi}$ and via $\Phi$ a representation of $\mathscr{H}$ with infinitesimal character $\chi$. It follows 
from Corollary 5.8 that $(\pi, V)$ is a hermitian or unitary representation of $\mathbb{H}_{\mathscr{Q}}$ precisely when it is a hermitian or unitary representation of $\mathscr{H}$.

We conclude this section with a few words on the relationship of hermitian representations and Theorem 3.3. The ${ }^{*}$ structure on the matrix algebra $\mathscr{M}_{n}$ in Theorem 3.3 is given by $E_{i, j}^{*}=E_{j, i}$. The irreducible module $\mathscr{C}_{n}$ of $\mathscr{M}_{n}$ admits a compatible positive definite hermitian form, namely,

$$
\langle v, w\rangle_{\mathscr{C}_{n}}=w^{*} \cdot v
$$

The functor $\mathscr{F}$ of Corollary 3.4 has the property that $\mathscr{V}$ admits a compatible hermitian form precisely when $\mathscr{F}(\mathscr{V})$ does. The two are related as follows. If $\mathscr{V}$ has invariant hermitian form $\langle$,$\rangle , then \mathscr{F}(\mathscr{V})$ has invariant hermitian form $\langle,\rangle_{\mathscr{F}}=\langle,\rangle_{\mathscr{C}_{n}} \cdot\langle$,$\rangle . Every *$ compatible invariant hermitian form on $\mathscr{F}(\mathscr{V})$ is a multiple of one of this type. The $\langle$,$\rangle is positive definite if and$ only if $\langle,\rangle_{\mathscr{F}}$ is positive definite.

\section{PARABOlic INDUCTION}

In this section, we relate the functor $\mathscr{F}$ of Corollary 3.4 with parabolic induction. We begin by recalling some preliminary facts. Let $\Pi_{M}$ be a subset of the simple roots $\Pi$ and let $P=M N \supset B$ be the corresponding parabolic subgroup and $\mathscr{I}_{M}=\mathscr{I} \cap M$. Denote by $W_{M}$ the subgroup of $W$ corresponding to $M$. Then the Iwahori Hecke algebra $\mathscr{H}\left(M / / \mathscr{J}_{M}\right)$ of $M$ is a subalgebra of $\mathscr{H}(G / / \mathscr{I})$. The algebra $\mathscr{H}(G / / \mathscr{I})$ is obtained from the generic Hecke algebra $\mathscr{H}$ by specializing the variable $z$ to $q$. The subalgebra $\mathscr{H}\left(M / / \mathscr{I}_{M}\right) \subset$ $\mathscr{H}(G / / \mathscr{I})$ is obtained from a subalgebra of $\mathscr{H}$ by the same specialization. Let $\mathscr{H}^{M}$ denote the generic Hecke algebra of $M$, and let $\mathscr{H}_{W_{M}}$ be the subalgebra of $\mathscr{H}_{W}$ generated by $T_{s_{\alpha}}\left(\alpha \in \Pi_{M}\right)$. Then,

$$
\mathscr{H}^{M} \equiv \mathscr{H}_{W_{M}} \otimes_{\mathbb{C}\left[z, z^{-1}\right]} \mathscr{A}\left[z, z^{-1}\right]
$$

A representation $V$ of $M$ is $\mathscr{I}_{M}$-spherical if it is admissible, of finite length, and if each submodule is generated by its $\mathscr{I}_{M}$-fixed vectors.

Theorem 6.1 (Bernstein).

$$
\left(\operatorname{Ind}_{P}^{G}(V)\right)^{\mathscr{I}}=\mathscr{H}(G / / \mathscr{I}) \otimes_{\mathscr{H}\left(M / / \mathscr{I}_{M}\right)} V^{\mathscr{I}_{M}} .
$$

The analogue of $(6.1)$ in $\mathbb{H}_{\mathscr{O}}$ is the subalgebra

$$
\mathbb{H}_{\mathscr{O}}^{M}=\mathbb{C} W_{M} \otimes\left(\mathscr{E} \otimes_{\mathbb{C}} \mathscr{S}[r]\right)
$$

If $V$ is a finite-dimensional $\mathbb{H}_{\mathscr{O}}^{M}$ module, we define the induced module $\operatorname{Ind}(V)$ to be

$$
\operatorname{Ind}(V)=\mathbb{H}_{\mathscr{O}} \otimes_{\mathbb{H}_{\mathscr{Q}}^{M}} V .
$$

Theorem 6.2. View $\mathscr{H}$ and $\mathbb{H}_{\mathscr{O}}$ as analytic families and recall $\Phi=\Phi_{\nu}$ from Theorem 4.3. Then the diagram 


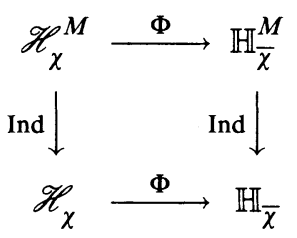

commutes.

Proof. This follows from Theorem 4.3 and the discussion above.

We recall some results on tempered representations. Let $V$ be a tempered representation (of $\mathscr{H}_{\chi}$ or equivalently of $\mathbb{H}_{\bar{\chi}}$ via the isomorphism $\Phi$ ). Since the subalgebra $\mathscr{S}[r] \subset \mathbb{H}_{\mathscr{O}}$ is abelian, there is a filtration

$$
\{0\}=V_{0} \subset V_{1} \subset V_{2} \subset \cdots \subset V_{d}=V \quad\left(\operatorname{dim}_{\mathbb{C}} V_{i}=i\right)
$$

such that each $V_{i}$ is $\mathscr{S}$ invariant and $\mathscr{S}$ acts by a character on $V_{i} / V_{i-1}(1 \leq$ $i \leq d)$. This character is completely determined by its restriction to $\mathscr{L}=$ $\mathbb{C} \otimes_{\mathbb{Z}} \mathscr{X} \subset \mathscr{S}$. Moreover, $\mathscr{L}$ can be viewed as the dual to the Lie algebra $\mathscr{L}=$ $\mathbb{C} \otimes_{\mathbb{Z}} \mathscr{Y}$ of ${ }^{L} A$. Thus it acts via a linear functional $\phi_{i} \in \check{\mathscr{L}}=\operatorname{Hom}_{\mathbb{C}}(\mathscr{L}, \mathbb{C})$ on $V_{i} / V_{i-1}(1 \leq i \leq d)$. The $\phi_{i}$ 's are called the generalized coweights of the action of $\mathscr{S}$ on $V$. If we write the infinitesimal character as $\chi=\left(e^{r}, e^{t}\right)$, then $e^{t}$ and $e^{\phi_{i}}$ are in the same Weyl group orbit. Let $\mathbb{F}$ denote either the real $\mathbb{R}$ or the complex $\mathbb{C}$ numbers, and set

$$
\begin{aligned}
\check{\mathscr{L}}_{R}(\mathbb{F}) & =\mathbb{F} \text {-span of simple roots } \check{\alpha} \text { in } \check{R} \\
\check{\mathscr{L}}_{R}^{\perp}(\mathbb{F}) & =\{v \in \mathscr{L}(\mathbb{F}) \mid\langle\alpha, v\rangle=0 \text { for all } \alpha \in R\} .
\end{aligned}
$$

Then $\check{\mathscr{L}}=\check{\mathscr{L}}_{R}(\mathbb{C}) \oplus \check{\mathscr{L}}_{R}^{\perp}(\mathbb{C})$. With the obvious notation, we write $i \check{\mathscr{L}}_{R}^{\perp}(\mathbb{R})$ for the imaginary elements in $\check{\mathscr{L}}_{R}^{\perp}(\mathbb{C})$. Set

$$
\begin{aligned}
\mathscr{C}= & \left\{y_{1}+y_{2} \mid y_{1} \in \check{\mathscr{L}}_{R}(\mathbb{C}), \quad y_{2} \in i \check{\mathscr{L}}_{R}^{\perp}(\mathbb{R}),\right. \\
& \text { and } \left.\operatorname{Re}\left(\left\langle y_{1}, \omega\right\rangle\right)<0 \text { for } \omega \text { a fundamental weight }\right\}, \\
\overline{\mathscr{C}}= & \left\{y_{1}+y_{2} \mid y_{1} \in \check{\mathscr{L}}_{R}(\mathbb{C}), \quad y_{2} \in i \check{\mathscr{L}}_{R}^{\perp}(\mathbb{R}),\right. \\
& \text { and } \left.\operatorname{Re}\left(\left\langle y_{1}, \omega\right\rangle\right) \leq 0 \text { for } \omega \text { a fundamental weight }\right\} .
\end{aligned}
$$

A restatement of the condition on $y_{1}$ is that

$$
y_{1} \in \sum_{\check{\alpha} \in \check{R}^{+}} c_{\alpha} \otimes \check{\alpha}, \quad \text { where } \operatorname{Re}\left(c_{\alpha}\right)<0 \text { for } y_{1} \in \mathscr{C}\left(\operatorname{Re}\left(c_{\alpha}\right) \leq 0 \text { for } y_{1} \in \overline{\mathscr{C}}\right) .
$$

Suppose $(\pi, V)$ is an irreducible $\mathscr{I}$-spherical representation of $G$, such that $V^{\mathscr{I}}$ has infinitesimal character $\chi$.

Theorem 6.3 (Casselman). The representation $(\pi, V)$ is tempered (resp. discrete series) if and only if all the coweights $\phi_{i}$ of $V^{\mathscr{F}}$ lie in $\overline{\mathscr{C}}$ (resp. $\mathscr{C}$ ).

Theorem 6.4. (1) Suppose $V$ is a module for $\mathbb{H}_{\mathscr{Q}}^{M}$ and $\left\{\phi_{i}\right\}$ are the coweights of $V$. Let $\left\{w_{1}=1, w_{2}, \ldots, w_{n}\right\}$ be the coset representatives of $W / W_{M}$ of minimal length. Then $\left\{w_{j} \phi_{i}\right\}$ are the coweights of $\operatorname{Ind}(V)$. 
(2) Suppose $\sigma \in \mathscr{O}, V$ is a module for $\Gamma_{\sigma} \mathbb{H}_{\sigma}$, and $\left\{\phi_{i}\right\}$ are the coweights of $V$. Let $\left\{w_{1}=1, w_{2}, \ldots, w_{n}\right\}$ be the coset representatives of $W / \operatorname{Stab}(\sigma)$ of minimal length. Then $\left\{w_{j} \phi_{i}\right\}$ are the coweights of $\mathscr{F}(V)$.

Proof. The proof of case (1) and (2) are essentially the same. We give the details only in the case of parabolic induction. Let $\{0\}=V_{0} \subset V_{1} \subset V_{2} \subset \cdots \subset V_{d}=V$ be as in (6.5). For each $i>0$ choose a vector $v_{i} \in V_{i}-V_{i-1}$. The set $\left\{t_{w_{k}} \otimes v_{i}\right\}$ is a basis for $\operatorname{Ind}(V)$ (over $\mathbb{C}$ ).

Arrange the coset representatives $w_{j}$ 's so that $\ell\left(w_{j}\right) \leq \ell\left(w_{j+1}\right)$. Let $\prec$ be the lexicographical ordering on the pairs $(j, i)(1 \leq j \leq n, 1 \leq i \leq d)$, i.e.,

$$
(j, i) \prec(k, l) \text { if } j \leq k \text { or } j=k \text { and } i \leq l \text {, }
$$

and set

$$
V_{(i, j)}=\text { span of all } w_{k} \otimes v_{l} \text { with }(k, l) \preceq(j, i) .
$$

If $(1,0) \prec(j, i)$, let

$$
(j, i)^{\prime}= \begin{cases}(j, i-1) & \text { if } i>0 \\ (j-1, d) & \text { if } i=0\end{cases}
$$

Then $V_{(j, i)} / V_{(j, i)^{\prime}}$ is one dimensional generated by the image of $w_{j} \otimes v_{i}$. Relation (3.8) shows that in general

$$
\omega t_{w}=w(\omega) t_{w}+\sum_{x \prec w} t_{x} \omega_{x}
$$

where $\prec$ is the Bruhat order. In particular, $l(x)<l(w)$, so by $(6.5)$,

$$
\omega\left(t_{w_{j}} \otimes v_{i}\right)=w_{j}\left(\phi_{i}\right)(\omega) w_{j} \otimes v_{i}\left(\bmod V_{(j, i)^{\prime}}\right)
$$

for $\omega \in \mathscr{L}$.

Corollary 6.5. If $V$ is a tempered module for $\mathbb{H}_{\mathscr{\theta}}^{M}$ (resp. $\left.\Gamma_{\sigma} \mathbb{H}_{\sigma}\right)$, then $\operatorname{Ind}(V)$ (resp. $\mathscr{F}(V)$ ) is a tempered module of $\mathbb{H}_{\mathscr{O}}$.

Proof. In the case of parabolic induction, let $R_{M}^{+}$be the positive roots of $M$. For $W^{\prime}=W_{M}$ (resp. $\left.W_{\sigma}\right)$, let $R^{\prime+}$ be $R_{M}^{+}$(resp. $\left.R_{\sigma}^{+}\right)$. The representatives $\left\{w_{i}\right\}$ of $W / W^{\prime}$ of minimal length are the elements

$$
\left\{w_{i}\right\}=\left\{w \in W \mid w^{-1}\left(R^{\prime+}\right) \subset R^{+}\right\} .
$$

The corollary follows from this characterization of the minimal length elements.

We recast the Langlands classification in terms of the graded algebra $\mathbb{H}_{\mathscr{O}}$. We assume $r$ has been specialized to $\log \left(q^{1 / 2}\right)$. For $\Pi_{M} \subset \Pi$, let $\mathscr{L}=$ $\mathscr{L}_{R_{M}}(\mathbb{C}) \oplus \mathscr{L}_{R_{M}}^{\perp}(\mathbb{C})$ where $\mathscr{L}_{R_{M}}(\mathbb{C})$ and $\mathscr{L}_{R_{M}}^{\perp}(\mathbb{C})$ are defined, with the obvious change in notation, as in (6.6). Denote by $\mathscr{S}_{R_{M}}$ (resp. $\mathscr{S}_{R_{M}}^{\perp}$ ) the symmetric algebra of $\mathscr{L}_{R_{M}}(\mathbb{C})$ (resp. $\mathscr{L}_{R_{M}}^{\perp}(\mathbb{C})$ ). The symmetric algebra $\mathscr{S}$ is then the 
algebra tensor product $\mathscr{S}=\mathscr{S}_{R_{M}} \otimes_{\mathbb{C}} \mathscr{S}_{R_{M}}^{\perp}$. Then $\mathscr{S}_{R_{M}}^{\perp}$ commutes with the subalgebra

$$
B=\mathbb{C} W_{M} \otimes \mathscr{S}_{R_{M}}[r]
$$

and $\mathbb{H}_{\mathscr{O}}^{M}$ decomposes as

$$
\mathbb{H}_{\mathscr{O}}^{M}=B \otimes_{\mathbb{C}} \mathscr{S}_{R_{M}}^{\perp}
$$

Each $\nu \in \check{\mathscr{L}}_{R_{M}}(\mathbb{C})$ defines an algebra homomorphism $\phi_{\nu}: \mathscr{S}_{R_{M}}^{\perp} \longrightarrow \mathbb{C}$. If $(\pi, V)$ is a $\mathbb{H}_{\mathscr{O}}^{M}$-module, define $\left(\pi_{n} u, V_{\nu}\right)$ to be the $\mathbb{H}_{\mathscr{O}}^{M}$-module whose space is $V$ with the action

$$
\pi_{\nu}(h \cdot \lambda) v=\phi_{\nu}(\lambda) \pi(h \cdot \lambda) v,
$$

where $h \in B$ and $\lambda \in \mathscr{S}_{R_{M}}^{\perp}$. This construction is the analogue of twisting a representation of $M \subset G$ by a one-dimensional character of $M$.

Given data $(M, V, \nu)$ such that $V$ is tempered irreducible, define the standard module $X(V, \nu)$ to be $\operatorname{Ind}\left(\pi_{\nu}, V_{\nu}\right)$.

Theorem 6.6 (Langlands classification). (1) Suppose $V$ is an irreducible tempered module for $\mathbb{H}_{\mathscr{O}}^{M}$ and $\nu \in \check{\mathscr{L}}_{R_{M}}^{\perp}(\mathbb{R})$ with $\langle\alpha, \nu\rangle>0$ for all $\alpha \in R^{+}-R_{M}^{+}$. Then $X(V, \nu)=\operatorname{Ind}(V \otimes \nu)$ has a unique irreducible quotient $\bar{X}(V, \nu)$.

(2) Any irreducible module of $\mathbb{H}_{\mathscr{O}}$ occurs as some $\bar{X}(V, \nu)$. The data $(M, V, \nu)$ are unique (up to conjugation).

\section{COMPARISON}

We need to relate the functor $\mathscr{F}$ with parabolic induction. Let $s=s_{e} s_{h}$ be the polar decomposition of an element in ${ }^{L} A$ such that $\mathscr{O}=W \cdot s_{e}$ is finite. Write $s_{h}$ as $e^{\nu}$, where $\nu \in \check{\mathscr{X}} \otimes_{\mathbb{Z}} \mathbb{R}$. The centralizer ${ }^{L} M=C_{L_{G}}\left(s_{h}\right)$ of $s_{h}$ in ${ }^{L} G$ is obviously a Levi subgroup. The Weyl group $W_{M}$ of ${ }^{L} M$ is generated by the roots

$$
\Pi_{M}=\left\{\alpha \in \Pi \mid s_{\alpha}\left(s_{h}\right)=s_{h}\right\} .
$$

For $\sigma \in \mathscr{O}$, define $R_{\sigma}^{+} \subset R^{+}$and $R_{M, \sigma}^{+} \subset R_{M}^{+}$as in (3.12). Obviously,

$$
\text { if }\langle\nu, \alpha\rangle>0 \forall \alpha \in R^{+}-R_{M}^{+} \text {, then }\langle\nu, \alpha\rangle>0 \forall \alpha \in R_{\sigma}^{+}-R_{M, \sigma}^{+} \text {. }
$$

The orbit $\mathscr{O}$ decomposes under the action of $W_{M}$. These $W_{M}$-orbits in fact lead to a decomposition of $\mathbb{H}_{\mathscr{G}}^{M}$ into two-sided ideals. In order to formulate this decomposition we need to parametrize the $W_{M}$-orbits. This is accomplished by considering the double cosets $W_{M} \backslash W / \operatorname{Stab}\left(s_{e}\right)$. Suppose $w_{1}, w_{2}, \ldots, w_{k}$ are representatives for the double cosets. The desired parameterization is given by

$$
W_{M} w_{i} \operatorname{Stab}\left(s_{e}\right) \leftrightarrow \mathscr{O}_{i} \text { the } W_{M} \text {-orbit of } w_{i}\left(s_{e}\right) \text {. }
$$

Let $\tau_{i}=w_{i}\left(s_{e}\right)$. The stabilizer $\operatorname{Stab}\left(\tau_{i}\right)$ of $\tau_{i}$ in $W$ is $w_{i} \operatorname{Stab}\left(s_{e}\right) w_{i}^{-1}$. Likewise, the stablizer $W_{M, \tau_{i}}$ of $\tau_{i}$ in $W_{M}$ is $W_{M} \cap \operatorname{Stab}\left(\tau_{i}\right)$. It is the Weyl group of

$$
{ }^{L} M_{i}={ }^{L} M \cap C_{L_{G}}\left(\tau_{i}\right)=\operatorname{Cent}\left(\tau_{i},{ }^{L} M\right) .
$$


In particular,

$$
\left|\mathscr{O}_{i}\right|=\left[W_{M}: W_{M, \tau_{i}}\right]=\left[W_{M} w_{i} \operatorname{Stab}\left(s_{e}\right): \operatorname{Stab}\left(s_{e}\right)\right] .
$$

Let $\mathscr{H}^{M_{i}}$ be the Hecke algebra associated to ${ }^{L} M_{i}$. Then we can form the graded algebras $\mathbb{H}_{\mathscr{O}_{i}}^{M}$ and $\mathbb{H}_{i}^{M} \cong \mathbb{H}_{\tau_{i}}^{M}$ and $\left(\mathbb{H}_{\tau_{i}}^{M}\right)^{\prime}$ as in $\S 3$.

Proposition 7.1. (1) For each orbit $\mathscr{O}_{i}$,

$$
\mathbb{H}_{\mathscr{O}_{i}}^{M}=\mathbb{C} W_{M} \otimes_{\mathbb{C}}\left(\bigoplus_{\tau \in \mathscr{O}_{i}} \mathbb{C} E_{\tau}\right) \otimes_{\mathbb{C}} \mathscr{S}[r]
$$

is a two-sided ideal of $\mathbb{H}_{\mathscr{O}}^{M}$. It is isomorphic to $\left(\mathbb{H}_{\tau_{i}}^{M}\right)^{\prime} \otimes M_{n_{i}}(\mathbb{C})$.

(2) The ideals $\mathbb{H}_{\mathscr{O}_{i}}^{M}$ commute and

$$
\mathbb{H}_{\mathscr{O}}^{M}=\bigoplus_{i} \mathbb{H}_{\mathscr{O}_{i}}^{M}
$$

Proof. The fact that $\mathbb{H}_{\mathscr{O}_{i}}^{M}$ are commuting ideals and the decomposition in (2) follow from the commutation relations (3.3) between the $E_{\tau}$ 's. The isomorphism

$$
\mathbb{H}_{\mathscr{O}_{i}}^{M} \cong\left(\mathbb{H}_{\tau_{i}}^{M}\right)^{\prime} \otimes M_{n_{i}}(\mathbb{C})
$$

is Theorem (3.3) applied to this setting.

We now apply these results to compare Langlands standard modules for the Hecke algebras $\mathbb{H}_{\mathscr{O}}$ and $\mathbb{H}_{s_{e}}$.

Write ${ }^{L} G_{i}$ for the group $w_{i}{ }^{L} G\left(s_{e}\right) w_{i}^{-1}$, where ${ }^{L} G\left(s_{e}\right)$ is the stabilizer of $s_{e}$. It is important to note that ${ }^{L} M_{i}$ is a Levi subgroup of ${ }^{L} G_{i}$; indeed, it is the centralizer of $w_{i}^{-1} s_{h} w_{i}$. Thus, given a module $V^{\prime}$ of $\left(\mathbb{H}_{\tau_{i}}^{M}\right)^{\prime}$ we have two ways to form a module of $\mathbb{H}_{\mathscr{O}}$.

One way is to induce $V^{\prime}$ from $\left(\mathbb{H}_{\tau_{i}}^{M}\right)^{\prime}$ to $\left(\mathbb{H}_{\tau_{i}}\right)^{\prime}$ and then apply Theorem 3.3. This gives

$$
V_{1}=\left[\operatorname{Ind}_{\left(\mathbb{H}_{\tau_{i}}^{M}\right)^{\prime}}^{\mathbb{H}_{\tau^{\prime}}^{\prime}} V^{\prime}\right] \otimes_{\mathbb{C}} \mathscr{C}_{n}
$$

The other way is first to obtain a module $V^{\prime} \otimes_{\mathbb{C}} \mathscr{C}_{n_{i}}$ of $\mathbb{H}_{\mathscr{O}_{i}}^{M}$ via Proposition 7.1. Since $\mathbb{H}_{\mathscr{O}}^{M}$ is a two-sided ideal of $\mathbb{H}_{\mathscr{O}}^{M}$, we can view this module as a module for $\mathbb{H}_{\mathscr{Q}}^{M}$ and therefore induce it to $\mathbb{H}_{\mathscr{O}}$. This gives

$$
V_{2}=\operatorname{Ind}_{\mathbb{H}_{\mathscr{\theta}}^{M}}^{\mathbb{H}_{\mathscr{\theta}}}\left(V^{\prime} \otimes_{\mathbb{C}} \mathscr{C}_{n_{i}}\right)
$$

Theorem 7.2. The modules $V_{1}$ and $V_{2}$ of $\mathbb{H}_{\mathscr{Q}}$ obtained in this fashion are isomorphic. The isomorphism is compatible with *.

Proof. The modules $V_{1}$ and $V_{2}$ are spanned (over $\mathbb{H}_{\mathscr{Q}}$ ) by vectors of the form

$$
E_{1,1} \otimes_{\mathbb{H}_{\tau_{i}}}\left(h_{\tau_{i}} \otimes v\right) \quad\left(h_{\tau_{i}} \in \mathbb{H}_{\tau_{i}}\right)
$$


and

$$
1 \otimes_{\mathbb{H}_{\mathscr{O}}^{M}}\left(E_{1,1} \otimes v\right) \quad\left(h \in \mathbb{H}_{\mathscr{O}}, h^{\prime} \in \mathbb{H}_{\mathscr{O}}^{M}\right)
$$

respectively. Then the correspondence

$$
E_{1,1} \otimes_{\mathbb{H}_{\tau_{i}}}\left(h_{\tau_{i}} \otimes v\right) \leftrightarrow h_{\tau_{i}} \otimes_{\mathbb{H}_{\Theta}^{M}}\left(E_{1,1} \otimes_{\mathbb{H}_{\tau_{i}}^{\prime}}{ }^{M} v\right)
$$

defines an $\mathbb{H}_{\mathscr{O}}$ isomorphism between $V_{1}$ and $V_{2}$. This isomorphism is in fact a $*$ isomorphism.

We are now ready to compare standard modules.

Corollary 7.3. For $\nu \in \check{\mathscr{L}}_{R_{M}}^{\perp}$ satisfying $\langle\nu, \alpha\rangle>0$ for all $\alpha \in R^{+}-R_{M}^{+}$and $V$ tempered irreducible,

$$
X(V, \nu)=X\left(V^{\prime}, \nu\right) \otimes_{\mathbb{C}} \mathscr{C}_{n_{i}} .
$$

Proof. Suppose $V$ is an irreducible tempered module of $\mathbb{H}_{\mathscr{O}}^{M}$. By Proposition $7.1(2)$, there is a $W_{M}$-orbit $\mathscr{O}_{i} \subset \mathscr{O}$ such that $V$ is a representation of the ideal $\mathbb{H}_{\mathscr{O}_{i}}^{M}$. As a module of $\mathbb{H}_{\mathscr{O}_{i}}^{M}, V$ is tempered; so there is a tempered module $V^{\prime}$ of $\left(\mathbb{H}_{\tau_{i}}^{M}\right)^{\prime}$ so that $V=V^{\prime} \otimes_{\mathbb{C}} \mathscr{C}_{n_{i}}$. If $\nu \in \check{\mathscr{L}}_{R_{M}}^{\perp}$ satisfies the positivity conditions needed to form a standard module $X(V, \nu)$, then by $(7.1) X\left(V^{\prime}, \nu\right)$ is a standard module of $\left(\mathbb{H}_{\tau_{i}}\right)^{\prime}$.

\section{UNITARITY}

Let $G$ be a split reductive $p$-adic group. Our goal is Theorem 8.1. It shows that the unitarity of an Iwahori spherical representation of $G$ can be detected on the Iwahori fixed vectors. An easy reduction is that we need only consider the case when $G$ has connected center. This means the centralizer of any semisimple element $s \in{ }^{L} G$ is a connected reductive group.

Recall

$$
\bar{X}(V, \nu)=\text { unique irreducible quotient of } X(V, \nu)
$$

and that $\bar{X}$ admits a hermitian form if and only if there is a

$$
w \in \operatorname{Weyl}\left(G, \mathscr{Z}\left({ }^{L} M\right)\right)
$$

satisfying

$$
w(\nu)=-\nu \text { and } \quad V \approx w(V)
$$

There are two ways to place a hermitian form on the family of standard modules

$$
X_{t}=\text { Iwahori fixed vectors of } \operatorname{Ind}_{P}^{G}\left(V \otimes e^{t \nu}\right)(t \in \mathbb{R})
$$

they are:

(1) $\langle,\rangle_{t}$ : the form obtained from the standard intertwining operator

$$
\mathbb{L}: I(P, \sigma, \nu)=\operatorname{Ind}_{P}^{G}(V \otimes \nu) \rightarrow I(\bar{P}, \sigma, \nu)=\operatorname{Ind}_{\bar{P}}^{G}(V \otimes \nu) .
$$

(2) $\langle,\rangle_{t}^{\prime}$ : the form gotten, via Corollary 7.3, from the form on $X_{t}^{\prime}=$ $X\left(V^{\prime}, t \nu\right)$ and the isomorphism $X_{t}=X_{t}^{\prime} \otimes \mathscr{C}_{n}$. 
Both forms have the property that

$$
\left.\bar{X}_{t}=X_{t} / \text { radical of form }\right\} \quad(t>0),
$$

radical of form $=$ unique subrepresentation of $X_{t} \quad(t<0)$.

We conclude $\langle,\rangle_{t}$ and $\langle,\rangle_{t}^{\prime}$ differ by a memomorphic function which has no zeros or poles along the real axis.

Theorem 8.1. (1) $\bar{X}_{1}$ is unitary if and only if $\bar{X}_{1}^{\prime}$ is unitary.

(2) The unitarity of $\bar{X}_{1}$ is detected on the $\mathscr{I}$-fixed vectors.

Proof. It is obvious that the unitarity of the Iwahori fixed vectors of $\bar{X}_{1}$ is equivalent to the unitarity of the Iwahori fixed vectors of $\bar{X}_{1}^{\prime}$. For $G^{\prime}$, the group corresponding to ${ }^{L} G\left(s_{e}\right)$, we are in the situation of real infinitesimal character as in [BM]. Therefore, the unitarity of $\bar{X}_{1}^{\prime}$ is equivalent to the unitarity of its Iwahori fixed vectors. We need only show that $\bar{X}_{1}^{\prime}$ unitary implies $\bar{X}_{1}$ unitary. Let $\mathscr{T}\left(s_{e}\right)$ (resp. $\mathscr{T}^{\prime}\left(s_{e}\right)$ ) be the set of tempered representations of $G$ (resp. $G^{\prime}$ ) whose infinitesimal character has compact part $s_{e}$. Corollary 6.5 establishes a natural one-one map $\pi \leftrightarrow \pi^{\prime}$ between these two sets so that

$$
\begin{aligned}
\bar{X}_{1}^{ \pm} & =\sum_{\pi \in \mathscr{T}\left(s_{e}\right)} a_{\pi}^{ \pm} \theta_{\pi}^{K}, \\
\bar{X}_{1}^{\prime \pm} & =\sum_{\pi \in \mathscr{T}^{\prime}\left(s_{e}\right)} a_{\pi^{\prime}}^{ \pm} \theta_{\pi^{\prime}}^{K^{\prime}} \quad\left(a_{\pi^{\prime}}^{ \pm}=a_{\pi}^{ \pm}\right) .
\end{aligned}
$$

The unitarity of $\bar{X}_{1}^{\prime}$ means either $a_{\pi^{\prime}}^{+}$or $a_{\pi^{\prime}}^{-}$is identically zero for all $\pi^{\prime}$. This means $a_{\pi}^{+}$or $a_{\pi}^{-}$is identically zero for all $\pi$ and thus $\bar{X}_{1}$ is unitary.

\section{ACKNOWLEDGMENTS}

The authors thank George Lusztig for conversations on his work [Ls2, Ls3Ls4] and Andrei Zelevinski for some conversations on graded Hecke algebras.

\section{REFERENCES}

[B] D. Barbasch, The unitary dual for complex classical Lie groups, Invent. Math. 96 (1989), 103-176.

[BM] D. Barbasch and A. Moy, A unitarity criterion for p-adic groups, Invent. Math. 98 (1989), 19-38.

[BW] A. Borel and N. Wallach, Continuous cohomology, discrete subgroups and representations of reductive groups, Ann. of Math. Stud., vol. 94, Princeton Univ. Press, Princeton, NJ, 1980.

[KL] D. Kazhdan and G. Lusztig, Proof of the Deligne-Langlands conjecture for Hecke algebras, Invent. Math. 87 (1987), 153-215.

[Ls1] G. Lusztig, Some examples of square integrable representations of semisimple p-adic groups, Trans. Amer. Math. Soc. 277 (1983), 623-653.

[Ls2] G. Lusztig, Affine Hecke algebras and their graded version, J. Amer. Math. Soc. 2 (1989), 599-635. 
[Ls3] _ Cuspidal local systems and graded algebras. I, Inst. Hautes Étude Sci. Publ. Math. 67 (1988), 145-202.

[Ls4] _ Character sheaves. I-V, Adv. in Math. 56, 57, 59, 61 (1985-86).

[S] T. Springer, Reductive groups, Proc. Sympos. Pure Math., vol. 33, Amer. Math. Soc., Providence, RI, 1979, pp. 3-27.

[T] J. Tits, Reductive groups over local fields, Proc. Sympos. Pure Math., vol. 33, Amer. Math. Soc., Providence, RI, 1979, pp. 29-69.

[V] D. Vogan, Unitary representations of reductive Lie groups, Ann. of Math. Stud., vol. 118, Princeton Univ. Press, Princeton, NJ, 1987.

Department of Mathematics, Cornell University, Ithaca, New York 14853-7901

E-mail address: barbasch@math.cornell.edu

Department of Mathematics, University of Michigan, Ann Arbor, Michigan 481091003

E-mail address: moy@math.lsa.umich.edu 